\title{
EQUIVALENCE BETWEEN SUBSHRUBS AND CHAOTIC BANDS IN THE MANDELBROT SET
}

\author{
G. PASTOR, M. ROMERA, G. ALVAREZ, D. ARROYO, AND F. MONTOYA \\ Received 17 January 2006; Accepted 13 March 2006
}

We study in depth the equivalence between subshrubs and chaotic bands in the Mandelbrot set. In order to do so, we introduce the rules for chaotic bands and the rules for subshrubs, as well as the transformation rules that allow us to interchange them. From all the denominations of a chaotic band, we show the canonical form; that is, the one associated to the hyperbolic component that generates such a chaotic band. Starting from the study of the one-dimensional route, we fulfil an inductive study that gives a generalization of the shrub concept.

Copyright (c) 2006 G. Pastor et al. This is an open access article distributed under the Creative Commons Attribution License, which permits unrestricted use, distribution, and reproduction in any medium, provided the original work is properly cited.

\section{Introduction}

The Mandelbrot set [5,6] can be considered an archetype of what we call chaos. This set has already been widely studied by other authors $[1,3,4,8,17]$ always mathematicians since it is a mathematical object.

In this paper we try to study in depth the knowledge of the Mandelbrot set structure that was already treated by us in former papers [11-13, 19]. Although we are operating with a mathematical object, this paper has not been thought for mathematicians but for experimental scientists interested in the structure of the Mandelbrot set. Therefore, we do not use any demonstration nor theorem but the typical tools of experimental scientists. In this case, we use the measure of periods of hyperbolic components, and preperiods and periods of Misiurewicz points carried out in a large number of numerical experiments on this mathematical object. The outcomes of this paper are valid whereas no new "measure" shows the contrary, as admitted by experimental scientists.

We have used the Mandelbrot set profusely, more specifically its antenna, in order to see the one-dimensional (1D) quadratic maps [14]. Indeed, the intersection of the Mandelbrot set and the real axis is the real Mandelbrot set, described by the 1D quadratic map $x_{n+1}=x_{n}^{2}+c$. Since all the 1D quadratic maps are equivalent [9], the 1D quadratic map $x_{n}=x_{n+1}^{2}+c$ can be considered as representative of all the others. Therefore, we 
simply use the Mandelbrot set antenna (which has two dimensions) in order to see the real Mandelbrot set, or other real sets described by a 1D quadratic map (which have one dimension; i.e., they are segments).

In the already cited papers $[11-13,19]$, we have in some way followed the inverse process. We take advantage of the knowledge that we have got on the $1 \mathrm{D}$ quadratic maps structure [15] in order to study, starting from this knowledge, the Mandelbrot set structure. That is to say, in the same way that we use the Mandelbrot set in two dimensions in order to see the map $x_{n}=x_{n+1}^{2}+c$ in one dimension, we also use all we know about the structure of the map $x_{n}=x_{n+1}^{2}+c$ in one dimension, in order to study the Mandelbrot set structure, with two dimensions. We will see all this in Section 2, the structure of a 1D quadratic map in Section 2.1 and the structure of the Mandelbrot set in Section 2.2.

We had already studied the equivalence between shrubs and chaotic bands in the Mandelbrot set $[11-13,19]$. But taking into account the importance of the topic, this paper will focus on it and will study it in depth in Section 3. In order to do so, the equivalence will be shown more clearly and evidently, and, in addition, the rules to go from subshrubs to chaotic bands and vice versa will be introduced.

\section{Structures}

Let us begin by seeing the structure of a 1D quadratic map (as $x_{n+1}=x_{n}^{2}+c$ when the parameter value goes from $c=1 / 4$ to $c=-2$ that describes the real Mandelbrot set) and next the structure of the Mandelbrot set.

2.1. Structure of a 1D quadratic map. Let us remember the structure of a $1 \mathrm{D}$ quadratic map. Sharkovsky's ordering $[21,22]$ is the first important attempt to structure the chaos described by 1D quadratic maps. Sharkovsky's theorem [21, 22] gives a clear ordering of hyperbolic components, but only of hyperbolic components that appear the first time. For the case of $x_{n+1}=x_{n}^{2}+c$, this theorem states that the first appearance of the hyperbolic components is in the following universal ordering when the parameter value goes from $c=1 / 4$ to $c=-2$ :

$$
\begin{aligned}
1 & \prec 2 \prec 4 \prec 8 \cdots 2^{k} \cdot 9 \prec 2^{k} \cdot 7 \prec 2^{k} \cdot 5 \prec 2^{k} \cdot 3 \cdots 2 \cdot 9 \\
& \prec 2 \cdot 7 \prec 2 \cdot 5 \prec 2 \cdot 3 \cdots 9 \prec 7 \prec 5 \prec 3,
\end{aligned}
$$

where the symbol $\prec$ must be read as "precedes." Although this is only an ordering of the first appearance hyperbolic components (i.e., also an ordering of the natural numbers) it strongly contributes to the structure of 1D quadratic maps. Indeed, $1 \prec 2 \prec 4 \prec 8 \cdots$ are the ordered periods of the well-known period-doubling cascade, $\cdots 2^{k} \cdot 9 \prec 2^{k} \cdot 7 \prec 2^{k}$. $5 \prec 2^{k} \cdot 3$ are the periods of the first appearance hyperbolic components of the period$2^{k}$ chaotic band $\mathbf{B}_{k}, \cdots 2 \cdot 9 \prec 2 \cdot 7 \prec 2 \cdot 5 \prec 2 \cdot 3$ are the periods of the first appearance hyperbolic components of the period- $2^{1}$ chaotic band $\mathbf{B}_{1}$, and $\cdots 9 \prec 7 \prec 5 \prec 3$ are the periods of the first appearance hyperbolic components of the period- $2^{0}$ chaotic band $\mathbf{B}_{0}$.

We have introduced the "harmonic structure" of a 1D quadratic map [15]. In this harmonic structure one has on the one hand the period-doubling cascade, which is the 


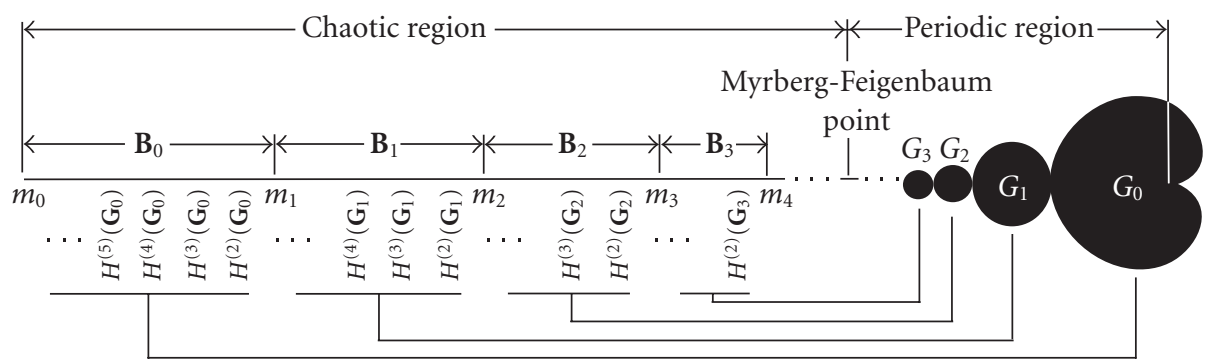

Figure 2.1. Sketch of the real Mandelbrot set, corresponding to the 1D quadratic map $x_{n}=x_{n+1}^{2}+c$, by using the neighborhood of the real part of the Mandelbrot set. Both the separation of periodic and chaotic regions, and the correspondence between the gene $\mathbf{G}_{i}$ and the chaotic band $\mathbf{B}_{i}$ are shown.

periodic region, and on the other hand the chaotic bands, which constitute the chaotic region, both separated by the Myrberg-Feigenbaum point [15]. As can be seen in [15], starting from the symbolic sequence $[7,16,20,23]$ of the $n$th hyperbolic component of the period-doubling cascade, the gene $\mathbf{G}_{n}$ whose period is $2^{n}$, one can calculate the symbolic sequences of the last appearance hyperbolic components of the chaotic band $\mathbf{B}_{n}$. These last appearance hyperbolic components, that we call structural components, constitute the true structure of a 1D quadratic map since, as we saw in our paper about heredity [18], starting from them one can calculate all the others.

Figure 2.1 clearly shows the periodic and chaotic regions separated by the MyrbergFeigenbaum point. Likewise it shows the correspondence between each hyperbolic component of the period-doubling cascade $\mathbf{G}_{i}(i=0,1,2, \ldots)$ and the chaotic band $\mathbf{B}_{i}(i=$ $0,1,2, \ldots)$. Calculations, that in [15] were done for symbolic sequences, in [10] have been done for external arguments. As can be seen in Figure 2.1, the Myrberg-Feigenbaum point can be considered as a "diabolic mirror" that makes to correspond the main cardioid $\mathbf{G}_{0}$, which is the last hyperbolic component on the right and has period 1 , with $\mathbf{B}_{0}$, which is the last chaotic band on the left and has period 1; the first disc of the period doubling cascade $\mathbf{G}_{1}$, which is the penultimate hyperbolic component on the right and has period 2 , with $\mathbf{B}_{1}$, which is the penultimate chaotic band on the left and has period 2; and so on. Diabolic mirror is that which transforms what is periodic in chaotic and vice versa.

If starting from the beginning of the periodic region, the cusp or the origin of the main cardioid in $c=0.25$, one wants to go up to the end of the chaotic region, the tip in $c=-2$, one only has a possible route because the $1 \mathrm{D}$ quadratic map is a segment. This only route has two parts: from $c=0.25$ to the MF point, which is the periodic route, and from the MF point to $c=-2$, which is the chaotic route. Taking into account the above cited correspondence, when the periodic route is covered, one is determining how the chaotic route will be.

2.2. Structure of the Mandelbrot set. We use the structure of the $1 \mathrm{D}$ quadratic map $x_{n+1}=x_{n}^{2}+c$ for $-2 \leq c \leq 1 / 4$, that has just been seen, in order to study the structure of the Mandelbrot set. Obviously, the structure of the Mandelbrot set is much more complex; however, both are similar in many aspects. Thus, now there are again a periodic 
region and a chaotic region. Nevertheless, even though in 1D quadratic maps there is only one point to separate both regions, now there are an infinity; that is, there are an infinity of Myrberg-Feigenbaum points.

Until now, to identify a primary hyperbolic component, which are those directly attached to the main cardioid, we used the rotation number $q_{1} / p_{1}[2,19]$, where $p_{1}$ indicates the hyperbolic component period and $q_{1}$ indicates the appearance order among the hyperbolic components with the same period. The rotation number of the main cardioid is $1 / 1,1$ in the denominator because the period is 1 , and 1 in the numerator because it is the first hyperbolic component-and the only one-with period 1 . One can think of the main cardioid as the only 0 -ary hyperbolic component, and therefore it would be $q_{0} / p_{0}$, where $q_{0} / p_{0}=1 / 1$. Then the primary hyperbolic components will be now $q_{0} / p_{0} \cdot q_{1} / p_{1}$ (or $\left.1 / 1 \cdot q_{1} / p_{1}\right)$, the secondary hyperbolic components $q_{0} / p_{0} \cdot q_{1} / p_{1} \cdot q_{2} / p_{2}$ (or $\left.1 / 1 \cdot q_{1} / p_{1} \cdot q_{2} / p_{2}\right), \ldots$, and the $N$-ary hyperbolic components $q_{0} / p_{0} \cdot q_{1} / p_{1} \cdots q_{N} / p_{N}$ (or $1 / 1 \cdot q_{1} / p_{1} \cdots q_{N} / p_{N}$ ). Sometimes $q_{0} / p_{0}$ (or $1 / 1$ ) is removed for convenience.

Starting from the main cardioid $1 / 1$, one can reach the chaos through an infinite number of periodic routes. To specify one definite periodic route, one has to specify the $N$-ary hyperbolic component through which the chaos is reached [13]. Indeed, as is known from [13], the periodic route has two parts: the generation route and the Myrberg-Feigenbaum route. The generation route is the route to reach the $N$-ary hyperbolic component $1 / 1$. $q_{1} / p_{1} \cdots q_{N} / p_{N}$ starting from the main cardioid $1 / 1$. This generation route consists of $N$ hyperbolic components: $1 / 1,1 / 1 \cdot q_{1} / p_{1}, 1 / 1 \cdot q_{1} / p_{1} \cdot q_{2} / p_{2}, \ldots, 1 / 1 \cdot q_{1} / p_{1} \cdots q_{N-1} / p_{N-1}$. The Myrberg-Feigenbaum route begins in the $N$-ary hyperbolic component and follows the hyperbolic components of its period doubling cascade up to the Myrberg-Feigenbaum point, which is the gate to reach chaos. Therefore, this Myrberg-Feigenbaum route consists of an infinity of hyperbolic components: $1 / 1 \cdot q_{1} / p_{1} \cdots q_{N} / p_{N}, 1 / 1 \cdot q_{1} / p_{1} \cdots q_{N} /$ $p_{N} \cdot 1 / 2,1 / 1 \cdot q_{1} / p_{1} \cdots q_{N} / p_{N} \cdot 1 / 2 \cdot 1 / 2, \ldots$. For convenience we denominate the periodic route to the chaos through the $N$-ary hyperbolic component $1 / 1 \cdot q_{1} / p_{1} \cdots q_{N} / p_{N}$ as follows: $1 / 1 \cdot q_{1} / p_{1} \cdots q_{N} / p_{N} \cdot \overline{1 / 2}$. If one states that $1 / 1 \cdot q_{1} / p_{1} \cdots q_{N} / p_{N} \cdot \overline{1 / 2}$ is a route, it means that the route followed is $1 / 1,1 / 1 \cdot q_{1} / p_{1}, 1 / 1 \cdot q_{1} / p_{1} \cdot q_{2} / p_{2}, 1 / 1 \cdot q_{1} / p_{1} \cdot q_{2} / p_{2}$. $q_{3} / p_{3}, \ldots, 1 / 1 \cdot q_{1} / p_{1} \cdots q_{N} / p_{N}, 1 / 1 \cdot q_{1} / p_{1} \cdots q_{N} / p_{N} \cdot 1 / 2,1 / 1 \cdot q_{1} / p_{1} \cdots q_{N} / p_{N} \cdot 1 / 2 \cdot$ $1 / 2, \ldots$, that finishes in $\operatorname{MF}^{(N)}\left(1 / 1 \cdot q_{1} / p_{1} \cdots q_{N} / p_{N}\right)$, which is the gate to chaos. Let us see that, since any route necessarily finishes in $\overline{1 / 2}$, it is not necessary to make $\overline{1 / 2}$ explicit, therefore it can be written or not. Thus, one can indistinctly put $\operatorname{MF}^{(N)}(1 / 1$. $\left.q_{1} / p_{1} \cdots q_{N} / p_{N} \cdot \overline{1 / 2}\right)$ or $\mathrm{MF}^{(N)}\left(1 / 1 \cdot q_{1} / p_{1} \cdots q_{N} / p_{N}\right)$. One can also indistinctly say route $1 / 1 \cdot q_{1} / p_{1} \cdots q_{N} / p_{N} \cdot \overline{1 / 2}$ or route through $1 / 1 \cdot q_{1} / p_{1} \cdots q_{N} / p_{N}$, and so on for other cases.

As can clearly be seen in Figure 2.2, on the one hand we call periodic route through $1 / 1 \cdot q_{1} / p_{1} \cdots q_{N} / p_{N}, \operatorname{pr}\left(1 / 1 \cdot q_{1} / p_{1} \cdots q_{N} / p_{N}\right)$, to the generation route of the hyperbolic component $1 / 1 \cdot q_{1} / p_{1} \cdots q_{N} / p_{N}$ followed by the Myrberg-Feigenbaum route of the hyperbolic component $1 / 1 \cdot q_{1} / p_{1} \cdots q_{N} / p_{N}$. Therefore,

$$
\operatorname{pr}\left(\frac{1}{1} \cdot \frac{q_{1}}{p_{1}} \cdots \frac{q_{N}}{p_{N}}\right)=\operatorname{gr}\left(\frac{1}{1} \cdot \frac{q_{1}}{p_{1}} \cdots \frac{q_{N}}{p_{N}}\right) \cup \operatorname{MFr}\left(\frac{1}{1} \cdot \frac{q_{1}}{p_{1}} \cdots \frac{q_{N}}{p_{N}}\right)
$$


If a Myrberg-Feigenbaum point is crossed, the chaotic region is reached. Shrubs [11$13,19]$ are used to represent this chaotic region. Shrubs are also split into two parts: the branch 0 , main branch or $\operatorname{shrub}_{0}\left(1 / 1 \cdot q_{1} / p_{1} \cdots q_{N} / p_{N}\right)$, and the shrub remainder, $\operatorname{shrub}_{r}\left(1 / 1 \cdot q_{1} / p_{1} \cdots q_{N} / p_{N}\right)$. That is to say

$$
\operatorname{shrub}\left(\frac{1}{1} \cdot \frac{q_{1}}{p_{1}} \cdots \frac{q_{N}}{p_{N}}\right)=\operatorname{shrub}_{0}\left(\frac{1}{1} \cdot \frac{q_{1}}{p_{1}} \cdots \frac{q_{N}}{p_{N}}\right) \cup \operatorname{shrub}_{r}\left(11 \cdot \frac{q_{1}}{p_{1}} \cdots \frac{q_{N}}{p_{N}}\right) .
$$

The $\operatorname{shrub}_{0}\left(1 / 1 \cdot q_{1} / p_{1} \cdots q_{N} / p_{N}\right)$ is formed by the infinite number of chaotic bands of the $N$-ary hyperbolic component $1 / 1 \cdot q_{1} / p_{1} \cdots q_{N} / p_{N}[11]$, that is to say

$$
\begin{aligned}
\operatorname{shrub}_{0} & \left(\frac{1}{1} \cdot \frac{q_{1}}{p_{1}} \cdots \frac{q_{N}}{p_{N}}\right) \\
& =\mathbf{B}_{0}\left(\frac{1}{1} \cdot \frac{q_{1}}{p_{1}} \cdots \frac{q_{N}}{p_{N}}\right) \cup \mathbf{B}_{1}\left(\frac{q_{1}}{p_{1}} \cdot \frac{q_{2}}{p_{2}} \cdots \frac{q_{N}}{p_{N}}\right) \cup \cdots \cup \mathbf{B}_{\infty}\left(\frac{1}{1} \cdot \frac{q_{1}}{p_{1}} \cdots \frac{q_{N}}{p_{N}}\right) \\
& =\bigcup_{i=0}^{\infty} \mathbf{B}_{i}\left(\frac{1}{1} \cdot \frac{q_{1}}{p_{1}} \cdots \frac{q_{N}}{p_{N}}\right) .
\end{aligned}
$$

Likewise, the $\operatorname{shrub}_{r}\left(1 / 1 \cdot q_{1} / p_{1} \cdots q_{N} / p_{N}\right)$ is constituted by $N$ subshrubs $[11-13,19]$. Hence,

$$
\begin{aligned}
\operatorname{shrub}_{r} & \left(\frac{1}{1} \cdot \frac{q_{1}}{p_{1}} \cdots \frac{q_{N}}{p_{N}}\right) \\
& =S_{1}\left(\frac{1}{1} \cdot \frac{q_{1}}{p_{1}} \cdots \frac{q_{N}}{p_{N}}\right) \cup S_{2}\left(\frac{1}{1} \cdot \frac{q_{1}}{p_{1}} \cdots \frac{q_{N}}{p_{N}}\right) \cup \cdots \cup S_{N}\left(\frac{1}{1} \cdot \frac{q_{1}}{p_{1}} \cdots \frac{q_{N}}{p_{N}}\right) \\
& =\bigcup_{i=0}^{N} S_{i}\left(\frac{1}{1} \cdot \frac{q_{1}}{p_{1}} \cdots \frac{q_{N}}{p_{N}}\right),
\end{aligned}
$$

where, for convenience, $\operatorname{subshrub}_{i}\left(1 / 1 \cdot q_{1} / p_{1} \cdots q_{N} / p_{N}\right)=S_{i}\left(1 / 1 \cdot q_{1} / p_{1} \cdots q_{N} / p_{N}\right)$.

As has just been seen, the periodic route is constituted by hyperbolic components, and it has two parts: the generation route, which has $N$ hyperbolic components, and the Myrberg-Feigenbaum route, which has an infinity of hyperbolic components. Likewise, the chaotic route is described by the shrubs that also have two parts: the shrub ${ }_{0}$, with an infinity of chaotic bands or subshrubs, and the shrub $r$, with $N$ subshrubs. We observe that, in the same way that in the one-dimensional case, now the periodic route and the chaotic route are related. Indeed, the infinite number of hyperbolic components of the Myrberg-Feigenbaum route are the gene from which the last appearance hyperbolic components_-or structural components — of the infinite chaotic bands of the $\operatorname{shrub}_{0}\left(1 / 1 \cdot q_{1} / p_{1} \cdots q_{N} / p_{N}\right)[12]$ can be calculated. As can clearly be seen in Figure 2.2, 


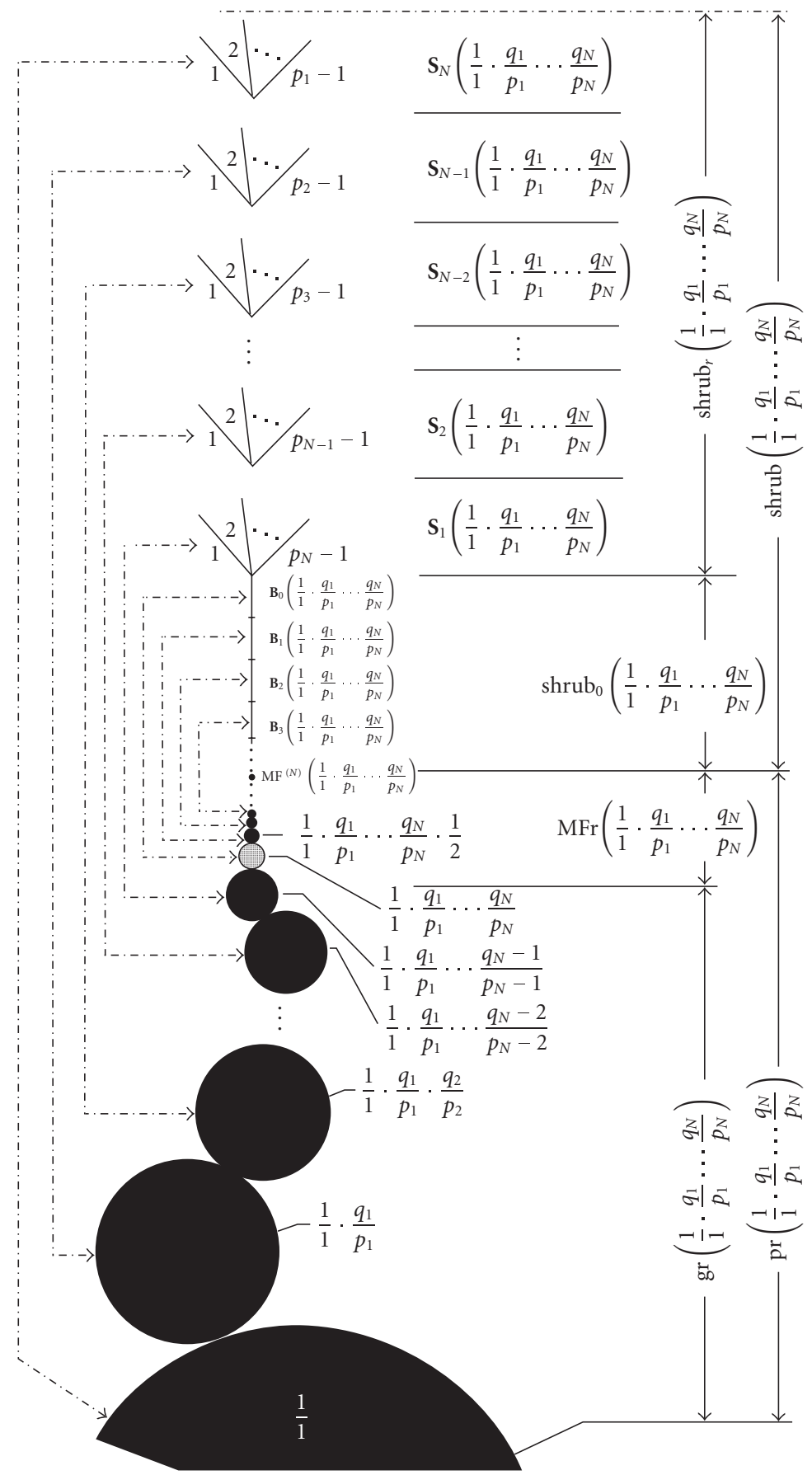

Figure 2.2. Sketch of the periodic route (constituted by the generation route and the MF route) followed by the corresponding chaotic route described by shrubs (constituted by the shrub 0 and the $\operatorname{shrub}_{r}$ ). The correspondence between elements of the periodic and chaotic routes are shown. 
the $\operatorname{shrub}_{0}\left(1 / 1 \cdot q_{1} / p_{1} \cdots q_{N} / p_{N}\right)$ seems the "diabolic image" of the $\operatorname{MFr}\left(1 / 1 \cdot q_{1} / p_{1} \cdots\right.$ $\left.q_{N} / p_{N}\right)$ in a mirror placed at $\operatorname{MF}^{(N)}\left(1 / 1 \cdot q_{1} / p_{1} \cdots q_{N} / p_{N}\right)$, as it happens in the onedimensional case.

Likewise, the $N$ hyperbolic components of the generation route are related with the $N$ subshrubs of the $\operatorname{shrub}_{r}\left(1 / 1 \cdot q_{1} / p_{1} \cdots q_{N} / p_{N}\right)[13]$ by means of the characteristic Misiurewicz points of the subshrub. Indeed, as can be seen in [13], 1/1 $\cdot q_{1} / p_{1} \cdots q_{N-i} / p_{N-i}$ is the gene of $S_{i}\left(1 / 1 \cdot q_{1} / p_{1} \cdots q_{N} / p_{N}\right)$, where $1 \leq i \leq N$. In Figure 2.2 the correspondence between $\operatorname{shrub}_{r}\left(1 / 1 \cdot q_{1} / p_{1} \cdots q_{N} / p_{N}\right)$ and $\operatorname{gr}\left(1 / 1 \cdot q_{1} / p_{1} \cdots q_{N} / p_{N}\right)$ has been clearly depicted. Also here $\operatorname{shrub}_{r}\left(1 / 1 \cdot q_{1} / p_{1} \cdots q_{N} / p_{N}\right)$ seems the specular image of $\operatorname{gr}\left(1 / 1 \cdot q_{1} / p_{1}\right.$ $\left.\cdots q_{N} / p_{N}\right)$ in a mirror placed at $\mathrm{MF}^{(N)}\left(1 / 1 \cdot q_{1} / p_{1} \cdots q_{N} / p_{N}\right)$. If each one of the two parts of the periodic route is the "diabolic image" of the corresponding part of the shrub (gr $\leftrightarrow \operatorname{shrub}_{r}$ and MFr $\leftrightarrow \operatorname{shrub}_{0}$ ) also the whole of the periodic route will be the "diabolic image" of the total of the chaotic route ( $\mathrm{pr} \leftrightarrow$ shrub). Therefore, when the periodic route is crossed, one is determining how the chaotic route will be.

Next we will focus on the object of this paper: the equivalence between subshrubs and chaotic bands. Since we have seen that they are equivalent, we have to establish the conditions under which the equivalence is given, and the rules under which subshrubs change to chaotic bands and vice versa. In order to do so, we are going to study some periodic routes and their corresponding chaotic routes or shrubs. As we normally do, we first analyze the one-dimensional case and then we extend it to the two-dimensional case.

\section{One-dimensional route}

In the one-dimensional case, the periodic route to reach the chaos starting from $1 / 1$ is $1 / 1 \cdot \overline{1 / 2}$. When the Myrberg-Feigenbaum point is crossed, the chaotic route is reached. Next we are going to analyze in some detail both routes.

The upper part of Figure 3.1 shows a sketch of the structural hyperbolic components and the notable points of these routes, showing them in a two-dimensional way to be able to see them. The periodic route consists of the main cardioid, $1 / 1$, and of the hyperbolic components corresponding to its period doubling cascade up to the MyrbergFeigenbaum point, where the chaotic route begins.

The lower part of Figure 3.1 is a table whose columns show the denomination of the chaotic bands depending on whether they come from the main cardioid $1 / 1$, from the first disc of the period doubling cascade $1 / 1 \cdot 1 / 2$, from the second disc $1 / 1 \cdot 1 / 2 \cdot 1 / 2$, from the third disc $1 / 1 \cdot 1 / 2 \cdot 1 / 2 \cdot 1 / 2$, or from the $i$ th disc $1 / 1(\cdot 1 / 2)^{i}$ (in the figure $1 / 1 \cdot \overline{1 / 2}^{i}$ due to space problems), as is explained as follows.

As is well known, the $2^{0}$-period chaotic band includes from the antenna tip, tip (1/1), that is the Misiurewicz point $M_{2,1}[9,14,15]$ placed on $c=-2$, to the band merging point $m_{1}=M_{3,1}$ placed on $c=-1.547 \ldots$ This band is called $\mathbf{B}_{0}(1 / 1)$, where the sub index, 0 , refers to the period of the chaotic band, $2^{0}$. We also adopted this notation because, in the one-dimensional case, the structural components of this chaotic band are the harmonics of the mentioned $1=2^{0}$-periodic main cardioid $1 / 1$, or $\mathbf{G}_{0}$ [15]. But sometimes, as will be seen next in the two-dimensional case, this notation is not a one-to-one notation because there are other chaotic bands with this same notation. In order to avoid this 


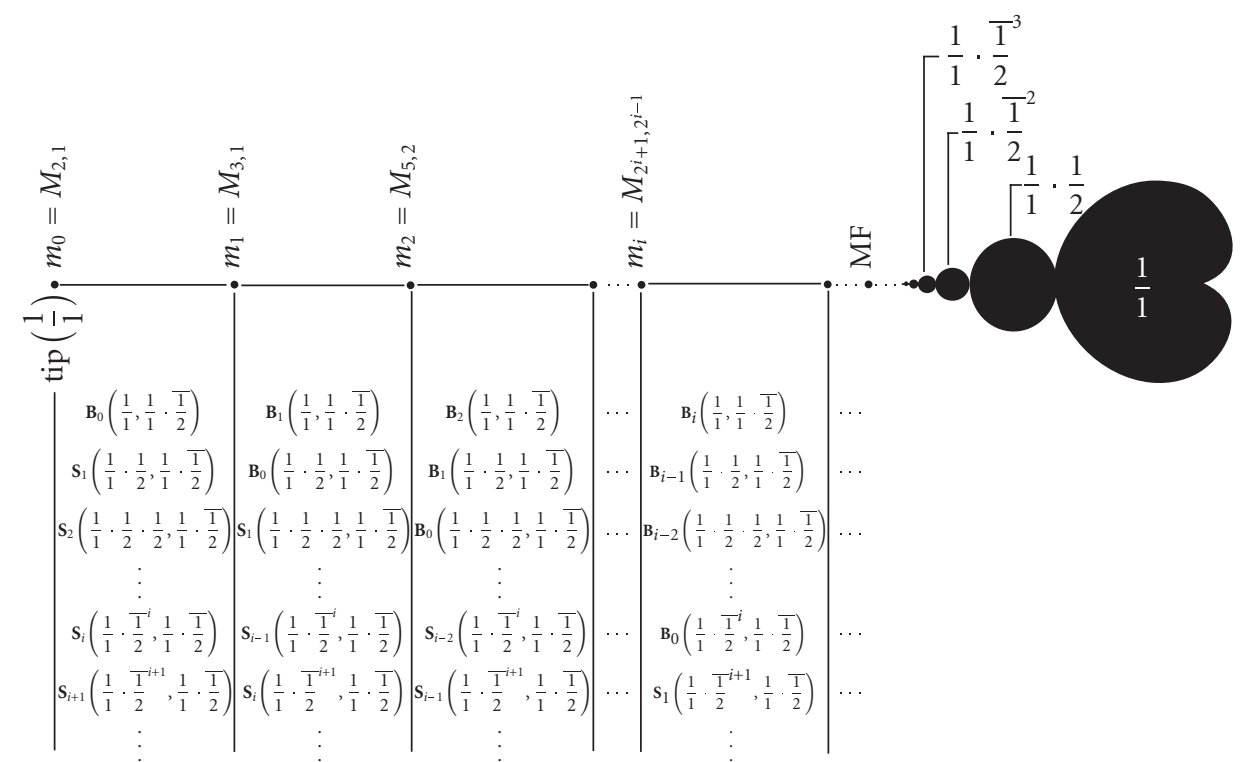

Figure 3.1. Sketch of the $1 \mathrm{D}$ route in a $2 \mathrm{D}$ representation in order to see it. The upper part shows the periodic and chaotic routes. The lower part shows a table with different names of each chaotic band.

problem, we call this chaotic band $\mathbf{B}_{0}(1 / 1,1 / 1 \cdot \overline{1 / 2})$ instead of simply calling it $\mathbf{B}_{0}(1 / 1)$. In $\mathbf{B}_{0}(1 / 1,1 / 1 \cdot \overline{1 / 2})$, in addition to the hyperbolic component that generates the chaotic band, the periodic route is given, both separated by a comma.

$\mathbf{B}_{0}(1 / 1,1 / 1 \cdot \overline{1 / 2})$ is the last band on the left, and therefore is the first column of the first row of the table of Figure 3.1. On its right, chaotic bands $\mathbf{B}_{1}(1 / 1,1 / 1 \cdot \overline{1 / 2}), \mathbf{B}_{2}(1 / 1,1 / 1$. $\overline{1 / 2}), \mathbf{B}_{3}(1 / 1,1 / 1 \cdot \overline{1 / 2}), \ldots, \mathbf{B}_{i}(1 / 1,1 / 1 \cdot \overline{1 / 2}), \ldots$, that finish in the MF point, are placed $[11-13,15,19]$. The first row represents $\operatorname{shrub}_{0}(1 / 1,1 / 1 \cdot \overline{1 / 2})$, and also $\operatorname{shrub}(1 / 1,1 / 1$. $\overline{1 / 2})$ because in this case $\operatorname{shrub}_{r}(1 / 1,1 / 1 \cdot \overline{1 / 2})=\phi$.

The $2^{1}$-periodic chaotic band extends from $m_{1}=M_{3,1}$ to $m_{2}=M_{5,2}$ [14]. This chaotic band is generated by the first disc of the period doubling cascade (which is the primary hyperbolic component $1 / 1 \cdot 1 / 2$ of period $2=2^{1}$ ) because in the one-dimensional case the structural components of this band are the harmonics of $1 / 1 \cdot 1 / 2$ [15]. In its simplified form the band is $\mathbf{B}_{0}(1 / 1 \cdot 1 / 2)$; but, according to what we said before, its complete form including the periodic route is $\mathbf{B}_{0}(1 / 1 \cdot 1 / 2,1 / 1 \cdot \overline{1 / 2})$. On its right the chaotic bands $\mathbf{B}_{1}(1 / 1 \cdot 1 / 2,1 / 1 \cdot \overline{1 / 2}), \mathbf{B}_{2}(1 / 1 \cdot 1 / 2,1 / 1 \cdot \overline{1 / 2}), \mathbf{B}_{3}(1 / 1 \cdot 1 / 2,1 / 1 \cdot \overline{1 / 2}), \ldots$, $\mathbf{B}_{i}(1 / 1 \cdot 1 / 2,1 / 1 \cdot \overline{1 / 2}), \ldots$ up to the MF point are placed. These chaotic bands constitute the $\operatorname{shrub}_{0}(1 / 1 \cdot 1 / 2,1 / 1 \cdot \overline{1 / 2})$. On the left of $\mathbf{B}_{0}(1 / 1 \cdot 1 / 2,1 / 1 \cdot \overline{1 / 2})$ the $\operatorname{shrub}_{r}(1 / 1 \cdot$ $1 / 2,1 / 1 \cdot \overline{1 / 2})$ is placed, which consists of only a subshrub, the $S_{1}(1 / 1 \cdot 1 / 2,1 / 1 \cdot \overline{1 / 2})$. All this can be seen in the second row of the table of Figure 3.1.

The $2^{2}$-periodic chaotic band extends from $m_{2}=M_{5,2}$ to $m_{3}=M_{9,4}$ [14]. This chaotic band, $\mathbf{B}_{0}(1 / 1 \cdot 1 / 2 \cdot 1 / 2,1 / 1 \cdot \overline{1 / 2})$, is generated by the secondary hyperbolic component $1 / 1 \cdot 1 / 2 \cdot 1 / 2$ because in the one-dimensional case the structural components of such a 
band are the harmonics of $1 / 1 \cdot 1 / 2 \cdot 1 / 2$. On its right the chaotic bands $\mathbf{B}_{1}(1 / 1 \cdot 1 / 2$. $1 / 2,1 / 1 \cdot \overline{1 / 2}), \mathbf{B}_{2}(1 / 1 \cdot 1 / 2 \cdot 1 / 2,1 / 1 \cdot \overline{1 / 2}), \ldots, \mathbf{B}_{i}(1 / 1 \cdot 1 / 2 \cdot 1 / 2,1 / 1 \cdot \overline{1 / 2}), \ldots$ up to the MF point are placed. These chaotic bands constitute the $\operatorname{shrub}_{0}(1 / 1 \cdot 1 / 2 \cdot 1 / 2,1 / 1 \cdot \overline{1 / 2})$. Since the generator is a secondary hyperbolic component, its shrub remainder has two subshrubs that are placed on the left, $\boldsymbol{S}_{1}(1 / 1 \cdot 1 / 2 \cdot 1 / 2,1 / 1 \cdot \overline{1 / 2})$ and $\mathbf{S}_{2}(1 / 1 \cdot 1 / 2 \cdot 1 / 2$, $1 / 1 \cdot \overline{1 / 2}$ ), as can be seen in the third row of the table of Figure 3.1.

Likewise, the $2^{3}$-periodic chaotic band extends from $m_{3}=M_{9,4}$ to $m_{4}=M_{17,8}$ [14]. This chaotic band, $\mathbf{B}_{0}(1 / 1 \cdot 1 / 2 \cdot 1 / 2 \cdot 1 / 2,1 / 1 \cdot \overline{1 / 2})$, is generated by the tertiary hyperbolic component $1 / 1 \cdot 1 / 2 \cdot 1 / 2 \cdot 1 / 2$. On its right the chaotic bands $\mathbf{B}_{1}(1 / 1 \cdot 1 / 2 \cdot 1 / 2$. $1 / 2,1 / 1 \cdot \overline{1 / 2}), \mathbf{B}_{2}(1 / 1 \cdot 1 / 2 \cdot 1 / 2 \cdot 1 / 2,1 / 1 \cdot \overline{1 / 2}), \ldots, \mathbf{B}_{i}(1 / 1 \cdot 1 / 2 \cdot 1 / 2 \cdot 1 / 2,1 / 1 \cdot \overline{1 / 2}), \ldots$ up to the MF point are placed. These chaotic bands constitute the $\operatorname{shrub}_{0}(1 / 1 \cdot 1 / 2$. $1 / 2 \cdot 1 / 2,1 / 1 \cdot \overline{1 / 2})$. Since the generator is a tertiary hyperbolic component, its shrub remainder has three subshrubs that are placed on the left, $S_{1}(1 / 1 \cdot 1 / 2 \cdot 1 / 2 \cdot 1 / 2,1 / 1 \cdot \overline{1 / 2})$, $S_{2}(1 / 1 \cdot 1 / 2 \cdot 1 / 2 \cdot 1 / 2,1 / 1 \cdot \overline{1 / 2})$, and $S_{3}(1 / 1 \cdot 1 / 2 \cdot 1 / 2 \cdot 1 / 2,1 / 1 \cdot \overline{1 / 2})$, that should be placed in the fourth row of the table of Figure 3.1 but they are not included due to space problems.

By generalizing, the $2^{i}$-periodic chaotic band extends from $m_{i}=M_{2^{i}+1,2^{i-1}}$ to $m_{i+1}=$ $M_{2^{i+1}+1,2^{i}}$ [14]. This chaotic band, $\mathbf{B}_{0}\left(1 / 1(\cdot 1 / 2)^{i}, 1 / 1 \cdot \overline{1 / 2}\right)$, is generated by the $i$-ary hyperbolic component $1 / 1(\cdot 1 / 2)^{i}$. On its right the chaotic bands $\mathbf{B}_{1}\left(1 / 1(\cdot 1 / 2)^{i}, 1 / 1 \cdot \overline{1 / 2}\right)$, $\mathbf{B}_{2}\left(1 / 1(\cdot 1 / 2)^{i}, 1 / 1 \cdot \overline{1 / 2}\right), \ldots, \mathbf{B}_{i}\left(1 / 1(\cdot 1 / 2)^{i}, 1 / 1 \cdot \overline{1 / 2}\right), \ldots$ up to the MF point are placed. These chaotic bands constitute the $\operatorname{shrub}_{0}\left(1 / 1(\cdot 1 / 2)^{i}, 1 / 1 \cdot \overline{1 / 2}\right)$. Since the generator is an $i$-ary hyperbolic component, its shrub remainder has $i$ subshrubs that are placed on the left, $\mathbf{S}_{1}\left(1 / 1(\cdot 1 / 2)^{i}, 1 / 1 \cdot \overline{1 / 2}\right), \ldots, \mathbf{S}_{i-1}\left(1 / 1(\cdot 1 / 2)^{i}, 1 / 1 \cdot \overline{1 / 2}\right)$ and $\mathbf{S}_{i}\left(1 / 1(\cdot 1 / 2)^{i}, 1 / 1 \cdot \overline{1 / 2}\right)$, as can be seen in the $(i+1)$-ary row of the table of Figure 3.1, although incompletely due to the figure size. In the lower part of Figure 3.1 we have given some data that will have a great utility. For example, one can see that a specific chaotic band can be named in many ways: all of the corresponding column. Since each column, that represents an only chaotic band, has both chaotic band names and subshrub names, the following statement can be given: subshrubs and chaotic bands have the same nature.

Let hc be the generic name of a hyperbolic component. As has been seen, we have called $\mathbf{B}_{i}$ (hc, route) the chaotic bands because it is needed to add the route to completely define the chaotic bands in the $2 \mathrm{D}$ case. The subshrubs can be called $\mathbf{S}_{i}$ (hc), because they are well defined without the route. However, although not necessary, we can call them $\mathbf{S}_{i}$ (hc, route) if uniformity is wanted.

Among all the ways we can call a chaotic band, one of them, that we call canonical form, is $\mathbf{B}_{0}$. This canonical form is the more important because the hyperbolic component associated to $\mathbf{B}_{0}$ is the generator of the chaotic band; that is, in the one-dimensional case that we are dealing with, the harmonics of such a generator are the structural components of the chaotic band [15]. The canonical form of a chaotic band is then $\mathbf{B}_{0}$ (generator, route). Therefore, when one is in the chaotic form one is relating the generator, which is a hyperbolic component of the periodic region, with the corresponding chaotic band, which is the chaotic band generated by such a generator. Hence, it is of great importance to calculate these canonical forms in order to know the relation between a chaotic band 
and the hyperbolic component of the periodic region that has generated such a chaotic band.

Let us see, in the case we are dealing with, how to find the canonical form of a chaotic band starting from a noncanonical form. In a first case, let us suppose that the noncanonical form is given as a subshrub. For example, let us suppose that one has $\boldsymbol{S}_{2}(1 / 1$. $1 / 2 \cdot 1 / 2,1 / 1 \cdot \overline{1 / 2})$. As can be seen in the first column, $S_{2}(1 / 1 \cdot 1 / 2 \cdot 1 / 2,1 / 1 \cdot \overline{1 / 2})=$ $\mathbf{S}_{1}(1 / 1 \cdot 1 / 2,1 / 1 \cdot \overline{1 / 2})=\mathbf{B}_{0}(1 / 1,1 / 1 \cdot \overline{1 / 2})$. Therefore, a subshrub with a specific index $i$ and a specific hc is the same as the subshrub with index $i-1$ and with an he which has lost the last fraction. As can be seen in Figure 3.1, when subshrubs indexes decrease, $\mathbf{S}_{1}(1 / 1 \cdot 1 / 2,1 / 1 \cdot \overline{1 / 2})$ should become $\mathbf{S}_{0}(1 / 1,1 / 1 \cdot \overline{1 / 2})$, but it became $\mathbf{B}_{0}(1 / 1,1 / 1 \cdot \overline{1 / 2})$. Hence $\mathbf{S}_{0}(1 / 1,1 / 1 \cdot \overline{1 / 2})=\mathbf{B}_{0}(1 / 1,1 / 1 \cdot \overline{1 / 2})$. When the index is zero the canonical form is reached. In this case the generator is the main cardioid $1 / 1$. Starting from $\mathbf{S}_{2}(1 / 1 \cdot 1 / 2 \cdot$ $1 / 2 \cdot 1 / 2,1 / 1 \cdot \overline{1 / 2})$, as can be seen in the second column, $S_{2}(1 / 1 \cdot 1 / 2 \cdot 1 / 2 \cdot 1 / 2,1 / 1 \cdot$ $\overline{1 / 2})=\mathbf{S}_{1}(1 / 1 \cdot 1 / 2 \cdot 1 / 2,1 / 1 \cdot \overline{1 / 2})=\mathbf{B}_{0}(1 / 1 \cdot 1 / 2,1 / 1 \cdot \overline{1 / 2})$, and therefore in this case the generator is the first disc of the period doubling cascade, $1 / 1 \cdot 1 / 2$.

Let us consider now the second case: the noncanonical form is given as a chaotic band. For example, let us consider $\mathbf{B}_{2}(1 / 1 \cdot 1 / 2,1 / 1 \cdot \overline{1 / 2})$. This chaotic band should be shown in Figure 3.1 in a fourth column, but it has already been said that it is not included due to figure size problems. Let us go to Table 3.1 that we will further explain. As can be seen in the fourth column, $\mathbf{B}_{2}(1 / 1 \cdot 1 / 2,1 / 1 \cdot \overline{1 / 2})=\mathbf{B}_{1}(1 / 1 \cdot 1 / 2 \cdot 1 / 2,1 / 1 \cdot \overline{1 / 2})=\mathbf{B}_{0}(1 / 1 \cdot 1 / 2$. $1 / 2 \cdot 1 / 2,1 / 1 \cdot \overline{1 / 2})$. Therefore, the chaotic band with index $i$ and a specific hyperbolic component he equals the chaotic band with index $i-1$ and an he which has increased one fraction, that is taken from the route. When the index is zero the canonical form is reached. In this case the generator is the third disc of the period doubling cascade $1 / 1 \cdot 1 / 2 \cdot 1 / 2 \cdot 1 / 2$.

We insist on something important that we said before: subshrubs and chaotic bands have the same nature. Therefore, if we carry out the appropriate transformations, we can work only with chaotic bands or only with subshrubs. To do so, we are going to introduce the following transformation rule:

$$
\mathbf{S}_{i}(\text { hc, route })=\mathbf{B}_{-i}(\text { hc, route }) .
$$

By applying this transformation rule to the table on the lower part of Figure 3.1, Table 3.1 is obtained if all the notations are expressed in chaotic bands, or Table 3.2 is obtained if all the notations are expressed in subshrubs.

Let us focus on Table 3.1. As we said before, all the notations of the same column are different names of the same chaotic band. Therefore, one can change from a name to another by following the following chaotic bands rule: one has to add (or remove) so many fractions as the number of units lost (or winned) by the index. That is to say, when the index increases the number of fractions decreases, and when the index decreases the number of fractions increases.

Let us notice now Table 3.2. Here all the notations of the same column are different names of the same subshrub. Hence, one can go from a name to another by following the following subshrubs rule: one has to add (or remove) so many fractions as the number of units winned (or lost) by the index. That is to say, now when the index increases the 
G. Pastor et al. 11

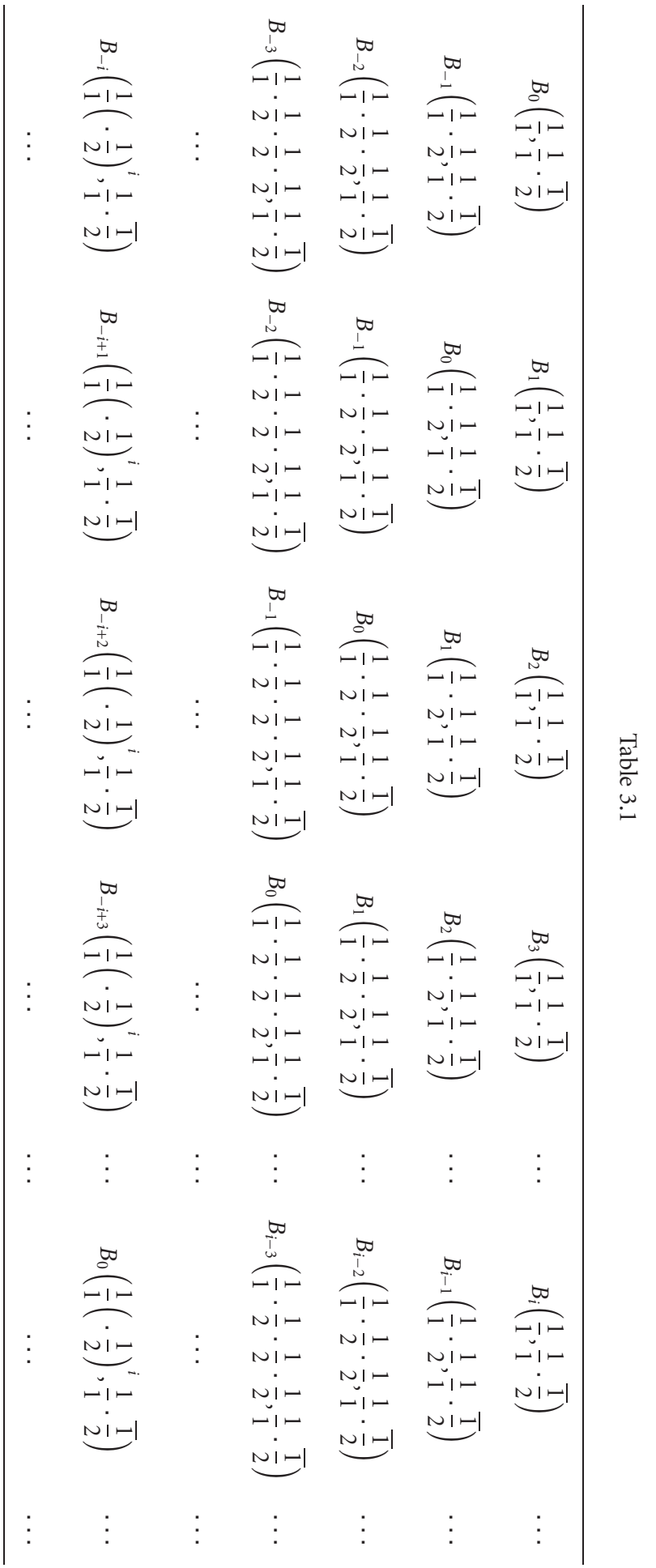


12 Subshrubs/chaotic bands equivalence in Mandelbrot set

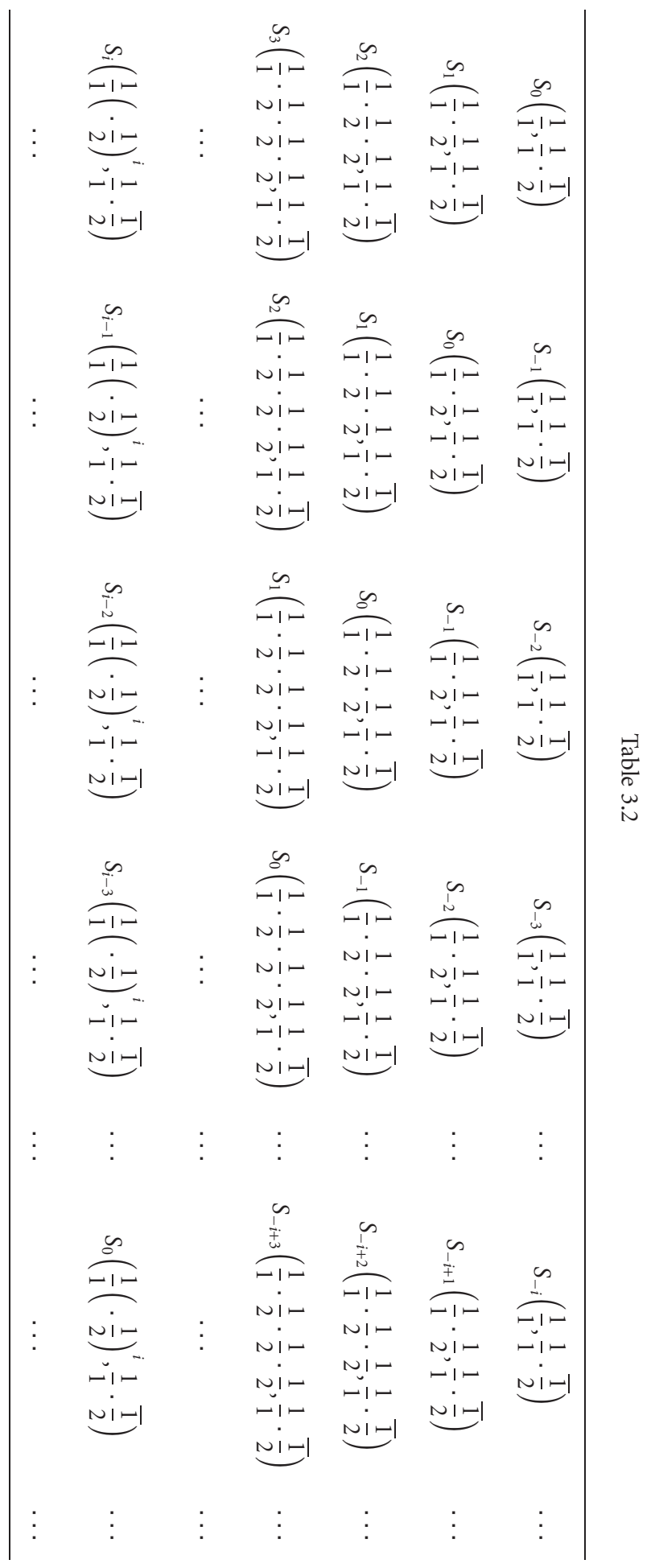


number of fractions also increases, and when the index decreases the number of fractions decreases.

If both Tables 3.1 and 3.2 are considered as matrices, the main diagonal is the canonical form of the different chaotic bands. Indeed, for example in Table 3.1, the main diagonal is $\mathbf{B}_{0}(1 / 1,1 / 1 \cdot \overline{1 / 2}), \mathbf{B}_{0}(1 / 1 \cdot 1 / 2,1 / 1 \cdot \overline{1 / 2}), \mathbf{B}_{0}(1 / 1 \cdot 1 / 2 \cdot 1 / 2,1 / 1 \cdot \overline{1 / 2}), \mathbf{B}_{0}(1 / 1 \cdot 1 / 2 \cdot 1 / 2 \cdot$ $1 / 2,1 / 1 \cdot \overline{1 / 2}), \ldots$, what means that the successive generators of the chaotic bands are $1 / 1$, $1 / 1 \cdot 1 / 2,1 / 1 \cdot 1 / 2 \cdot 1 / 2,1 / 1 \cdot 1 / 2 \cdot 1 / 2 \cdot 1 / 2, \ldots$, that just are the hyperbolic components of the periodic route that corresponds to the period doubling cascade.

\section{Primary route}

A route with periodic route generated by a primary hyperbolic component $1 / 1 \cdot q_{1} / p_{1}$, for example by the primary hyperbolic component of the Mandelbrot set $1 / 1 \cdot 1 / 3$, is called a primary route. In order to analyze this route, we will follow a similar process as the one followed in the periodic route $1 / 1 \cdot \overline{1 / 2}$ and its corresponding chaotic route seen before.

The periodic route starts from the main cardioid, $1 / 1$, and is followed by the primary hyperbolic component $1 / 1 \cdot 1 / 3$ and its period doubling cascade up to the MyrbergFeigenbaum point. Therefore, the periodic route is $1 / 1 \cdot 1 / 3 \cdot \overline{1 / 2}$. Next, the chaotic route, described by means of shrubs, is reached.

The upper part of Figure 4.1 shows a sketch of $\operatorname{shrub}(1 / 1 \cdot 1 / 3)$, or in its complete form $\operatorname{shrub}(1 / 1 \cdot 1 / 3,1 / 1 \cdot 1 / 3 \cdot \overline{1 / 2})$. This $\operatorname{shrub}(1 / 1 \cdot 1 / 3,1 / 1 \cdot 1 / 3 \cdot \overline{1 / 2})$ has two welldifferentiated parts: $\operatorname{shrub}_{0}(1 / 1 \cdot 1 / 3,1 / 1 \cdot 1 / 3 \cdot \overline{1 / 2})$ (constituted by the infinity of the chaotic bands of the hyperbolic component $1 / 1 \cdot 1 / 3)$ and $\operatorname{shrub}_{r}(1 / 1 \cdot 1 / 3,1 / 1 \cdot 1 / 3 \cdot$ $\overline{1 / 2}$ ) (constituted by $N$ subshrubs, that in this case is an only subshrub, $\boldsymbol{S}_{1}(1 / 1 \cdot 1 / 3,1 / 1$. $1 / 3 \cdot \overline{1 / 2}$ ), because $1 / 1 \cdot 1 / 3$ is a primary hyperbolic component. The first row of the table of the lower part of Figure 4.1 shows such a $\operatorname{shrub}(1 / 1 \cdot 1 / 3,1 / 1 \cdot 1 / 3 \cdot \overline{1 / 2})$ where, due to space problems, $g$ instead of $1 / 1 \cdot 1 / 3$ and pr instead of the periodic route $1 / 1 \cdot 1 / 3 \cdot \overline{1 / 2}$ are depicted. In the first column $\operatorname{shrub}_{r}(1 / 1 \cdot 1 / 3,1 / 1 \cdot 1 / 3 \cdot \overline{1 / 2})$ is given, or better said, its only subshrub $S_{1}(1 / 1 \cdot 1 / 3,1 / 1 \cdot 1 / 3 \cdot \overline{1 / 2})$. In the next columns the successive chaotic bands of $\operatorname{shrub}_{0}(1 / 1 \cdot 1 / 3,1 / 1 \cdot 1 / 3 \cdot \overline{1 / 2})$ are given: $\mathbf{B}_{0}(1 / 1 \cdot 1 / 3,1 / 1 \cdot 1 / 3 \cdot \overline{1 / 2}), \mathbf{B}_{1}(1 / 1 \cdot$ $1 / 3,1 / 1 \cdot 1 / 3 \cdot \overline{1 / 2}), \mathbf{B}_{2}(1 / 1 \cdot 1 / 3,1 / 1 \cdot 1 / 3 \cdot \overline{1 / 2}), \ldots, \mathbf{B}_{i}(1 / 1 \cdot 1 / 3,1 / 1 \cdot 1 / 3 \cdot \overline{1 / 2}), \ldots[11-$ $13,19]$.

The second row of the table of Figure 4.1 shows the shrub of the first disc of the period doubling cascade, which is the secondary hyperbolic component $1 / 1 \cdot 1 / 3 \cdot 1 / 2$ : $\operatorname{shrub}(1 / 1 \cdot 1 / 3 \cdot 1 / 2,1 / 1 \cdot 1 / 3 \cdot \overline{1 / 2})[11-13,19]$. The first and second columns show the two subshrubs of the shrub remainder, and the third and following columns show

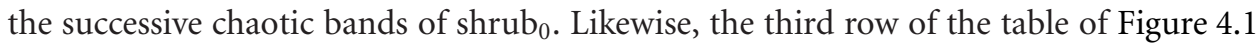
shows the $\operatorname{shrub}(1 / 1 \cdot 1 / 3 \cdot 1 / 2 \cdot 1 / 2,1 / 1 \cdot 1 / 3 \cdot \overline{1 / 2})$, which is the shrub of the second disc of the period doubling cascade, the tertiary hyperbolic component $1 / 1 \cdot 1 / 3 \cdot 1 / 2 \cdot 1 / 2$. The first, second, and third columns show the three subshrubs of the shrub remainder, and the fourth and following columns show the successive chaotic bands of the shrub $[11-13,19]$. Finally, the $i$ th row of the table of Figure 4.1 shows the shrub of the $i$-ary hyperbolic component $1 / 1 \cdot 1 / 3(\cdot 1 / 2)^{i-1}[11-13,19]$, which in the first $i$ columns has the 


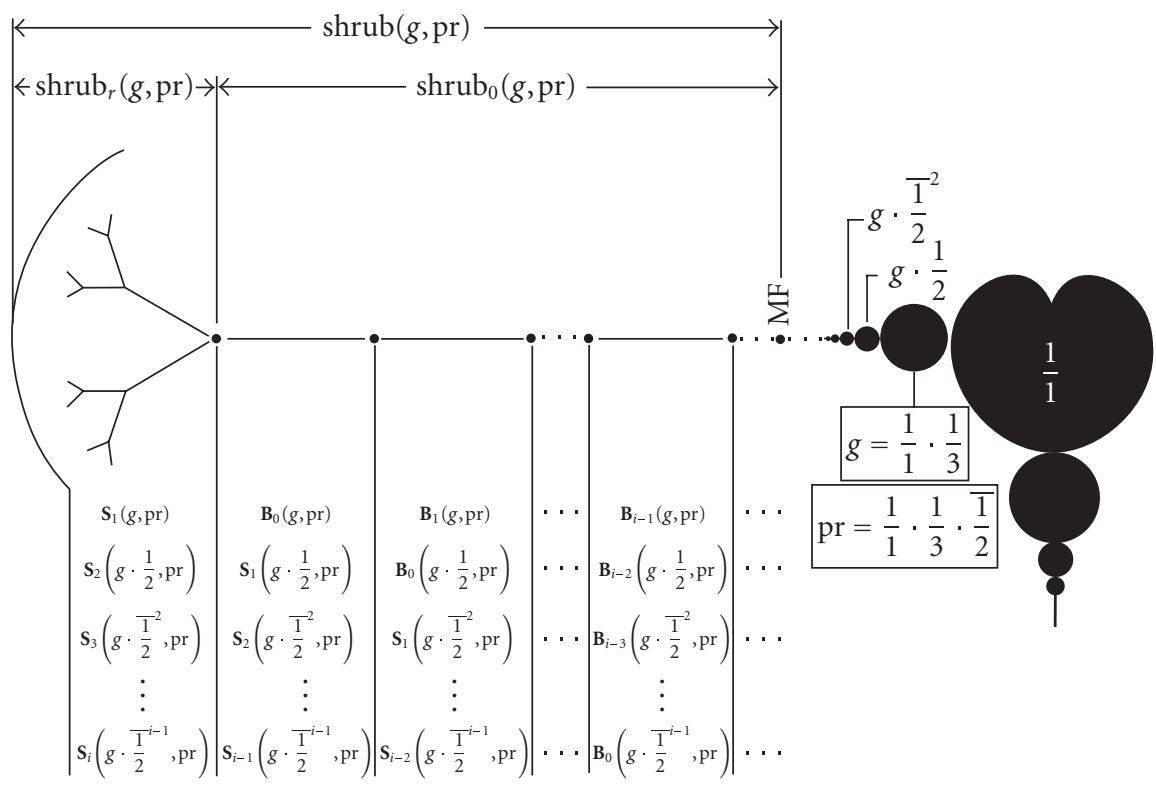

Figure 4.1. Sketch of the primary route through the primary hyperbolic component $1 / 1 \cdot 1 / 3$, showing the periodic route and the two parts of the chaotic route, $\operatorname{shrub}_{0}(1 / 1 \cdot 1 / 3,1 / 1 \cdot 1 / 3 \cdot \overline{1 / 2})$ and $\operatorname{shrub}_{r}(1 / 1 \cdot 1 / 3,1 / 1 \cdot 1 / 3 \cdot \overline{1 / 2})$. The lower part shows a table with different names of each chaotic band.

$i$ subshrubs of the shrub remainder, and next the successive chaotic bands of the shrub . The table is not as explicit as desirable due to the size of the figure.

Let us note the great analogy between the first row of Figure 4.1 and the second row of Figure 3.1. In Figure 3.1, as can be seen in the first column, the canonical form of $S_{1}(1 / 1 \cdot 1 / 2,1 / 1 \cdot \overline{1 / 2})$ is $\mathbf{B}_{0}(1 / 1,1 / 1 \cdot \overline{1 / 2})$. If one wants to see now the canonical form of $S_{1}(1 / 1 \cdot 1 / 3,1 / 1 \cdot 1 / 3 \cdot \overline{1 / 2})$ of Figure 4.1 , one cannot see it because it is not explicit in the figure. However, given the parallelism between both cases, one can find it by means of the shrub rule that says that to decrease the index one unit the last fraction has to be removed. According to that rule, $S_{1}(1 / 1 \cdot 1 / 3,1 / 1 \cdot 1 / 3 \cdot \overline{1 / 2})=S_{0}(1 / 1,1 / 1 \cdot 1 / 3 \cdot \overline{1 / 2})=$ $\mathbf{B}_{0}(1 / 1,1 / 1 \cdot 1 / 3 \cdot \overline{1 / 2})$. That is to say, the canonical forms of both $S_{1}(1 / 1 \cdot 1 / 2,1 / 1 \cdot \overline{1 / 2})$ and $S_{1}(1 / 1 \cdot 1 / 3,1 / 1 \cdot 1 / 3 \cdot \overline{1 / 2})$ have the same generator, which is the main cardioid $1 / 1$. However, both have different routes: in the first case the route is $1 / 1 \cdot \overline{1 / 2}$ and in the second case $1 / 1 \cdot 1 / 3 \cdot \overline{1 / 2}$. That is why we have made explicit the route in order to differentiate between both cases, as it was already noted at the beginning of Section 3.

When Figures 3.1 and 4.1 are carefully compared, the great analogy of the row $i$ ( $i=$ $1,2,3, \ldots)$ of Figure 4.1 and the row $i+1(i=1,2,3, \ldots)$ of Figure 3.1 can be seen. However, the first row of Figure 3.1 has not a similar row in Figure 4.1. In the former paragraph we calculated, by means of the shrubs rule, the first column of that nonexplicit row, and we obtained $\mathbf{S}_{0}(1 / 1,1 / 1 \cdot 1 / 3 \cdot \overline{1 / 2})=\mathbf{B}_{0}(1 / 1,1 / 1 \cdot 1 / 3 \cdot \overline{1 / 2})$. All the other columns of the row can be calculated by means of the chaotic bands rule. Table 4.1 is a repetition of the 
G. Pastor et al. 15

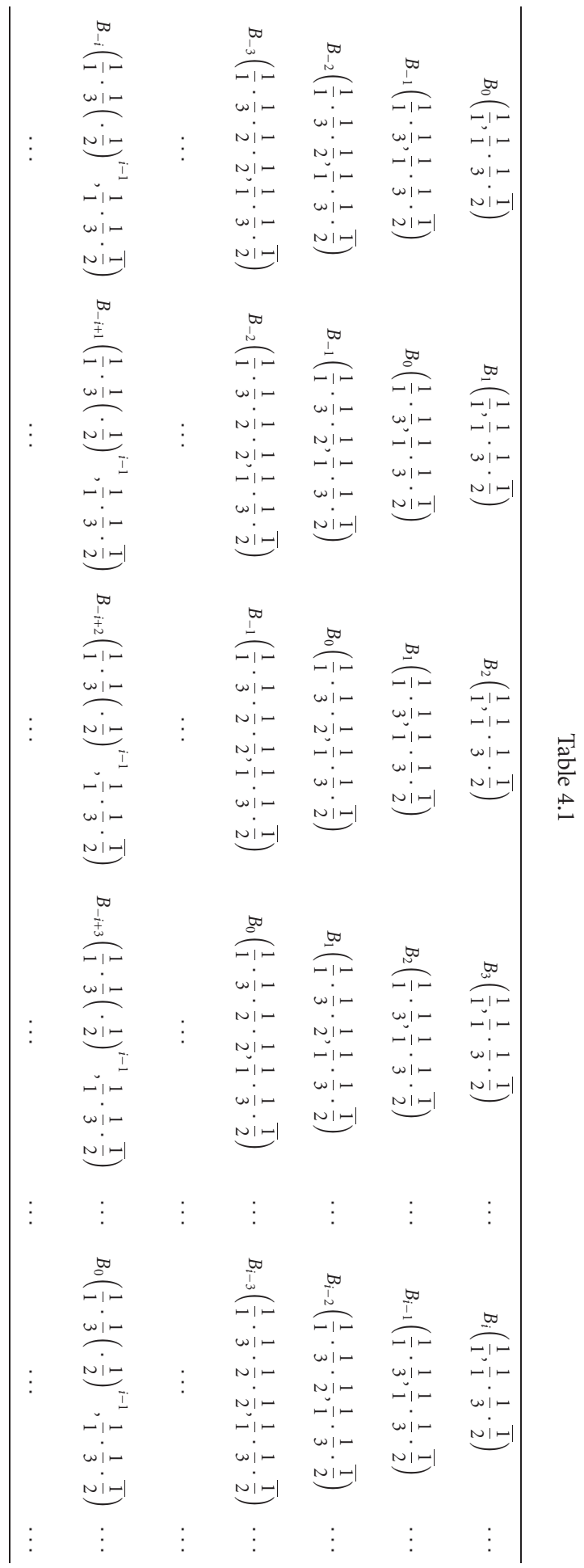


table of Figure 4.1 but with two modifications. First, the row that has just been calculated has been added as first row. Second, the transformation $S_{i}=B_{-i}$ has been used in order to give all the expressions in the form of chaotic bands. The above cited analogy between the tables of Figures 3.1 and 4.1 is now more evident between Tables 3.1 and 4.1. The results obtained for the route $1 / 1 \cdot 1 / 3 \cdot \overline{1 / 2}$ are parallel to those obtained for the route $1 / 1 \cdot \overline{1 / 2}$, with the same transformation as there and the same rules for chaotic bands and shrubs. If Table 4.1 is considered a matrix, again the main diagonal is the canonical form of the corresponding chaotic bands, therefore the successive chaotic band generators are $1 / 1,1 / 1 \cdot 1 / 3,1 / 1 \cdot 1 / 3 \cdot 1 / 2,1 / 1 \cdot 1 / 3 \cdot 1 / 2 \cdot 1 / 2, \ldots$, that are, again, the hyperbolic components of the periodic route followed now.

\section{Secondary and tertiary routes}

Let us begin by the secondary route. We call secondary route to a route whose generator is a secondary hyperbolic component $1 / 1 \cdot q_{1} / p_{1} \cdot q_{2} / p_{2}$, like $1 / 1 \cdot 1 / 3 \cdot 1 / 4$ that we will treat now. The periodic route starts from the main cardioid, $1 / 1$, and follows through the primary hyperbolic component $1 / 1 \cdot 1 / 3$ (both constitute the generation route). Next, one crosses the secondary hyperbolic component $1 / 1 \cdot 1 / 3 \cdot 1 / 4$ and the hyperbolic components of its period doubling cascade up to the Myrberg-Feigenbaum point (that constitute the Myrberg-Feigenbaum route). Then one reaches the chaotic route described by means of shrubs.

The upper part of Figure 5.1 shows the sketch of both the periodic route and the $\operatorname{shrub}(1 / 1 \cdot 1 / 3 \cdot 1 / 4,1 / 1 \cdot 1 / 3 \cdot 1 / 4 \cdot \overline{1 / 2})$. As it is known, the $\operatorname{shrub}_{0}(1 / 1 \cdot 1 / 3 \cdot 1 / 4,1 / 1 \cdot$ $1 / 3 \cdot 1 / 4 \cdot \overline{1 / 2})$ is constituted by the infinity of chaotic bands of the hyperbolic component $1 / 1 \cdot 1 / 3 \cdot 1 / 4$, and the $\operatorname{shrub}_{r}(1 / 1 \cdot 1 / 3 \cdot 1 / 4,1 / 1 \cdot 1 / 3 \cdot 1 / 4 \cdot \overline{1 / 2})$ is constituted by 2 subshrubs because it is a secondary hyperbolic component. The first row of the table of the lower part of Figure 5.1 shows such a $\operatorname{shrub}(1 / 1 \cdot 1 / 3 \cdot 1 / 4,1 / 1 \cdot 1 / 3 \cdot 1 / 4 \cdot \overline{1 / 2})$. $\operatorname{shrub}_{r}(1 / 1 \cdot 1 / 3 \cdot 1 / 4,1 / 1 \cdot 1 / 3 \cdot 1 / 4 \cdot \overline{1 / 2})$ is given in the first two columns, $S_{2}(1 / 1 \cdot 1 / 3 \cdot$ $1 / 4,1 / 1 \cdot 1 / 3 \cdot 1 / 4 \cdot \overline{1 / 2})$ in the first one and $S_{1}(1 / 1 \cdot 1 / 3 \cdot 1 / 4,1 / 1 \cdot 1 / 3 \cdot 1 / 4 \cdot \overline{1 / 2})$ in the second one. We have written $1 / 1 \cdot 1 / 3 \cdot 1 / 4 \cdot \overline{1 / 2}=g$ (gene) and $1 / 1 \cdot 1 / 3 \cdot 1 / 4 \cdot \overline{1 / 2}=$ $\mathrm{pr}$ (periodic route) in order to save space in the figure. The successive chaotic bands of the $\operatorname{shrub}_{0}(1 / 1 \cdot 1 / 3 \cdot 1 / 4,1 / 1 \cdot 1 / 3 \cdot 1 / 4 \cdot \overline{1 / 2}): \mathbf{B}_{0}(1 / 1 \cdot 1 / 3 \cdot 1 / 4,1 / 1 \cdot 1 / 3 \cdot 1 / 4 \cdot \overline{1 / 2})$, $\mathbf{B}_{1}(1 / 1 \cdot 1 / 3 \cdot 1 / 4,1 / 1 \cdot 1 / 3 \cdot 1 / 4 \cdot \overline{1 / 2}), \mathbf{B}_{2}(1 / 1 \cdot 1 / 3 \cdot 1 / 4,1 / 1 \cdot 1 / 3 \cdot 1 / 4 \cdot \overline{1 / 2}), \ldots, \mathbf{B}_{i}(1 / 1 \cdot$ $1 / 3 \cdot 1 / 4,1 / 1 \cdot 1 / 3 \cdot 1 / 4 \cdot \overline{1 / 2}), \ldots$, are given $[11-13,19]$ in the following columns.

The second row of the table of Figure 5.1 shows the shrub of the first disc of the period doubling cascade, which is the tertiary hyperbolic component $1 / 1 \cdot 1 / 3 \cdot 1 / 4 \cdot 1 / 2$ : the $\operatorname{shrub}(1 / 1 \cdot 1 / 3 \cdot 1 / 4 \cdot 1 / 2,1 / 1 \cdot 1 / 3 \cdot 1 / 4 \cdot \overline{1 / 2})[11-13,19]$. The three shrubs of the shrub remainder are shown in the first three columns, and the infinity of chaotic bands of the shrub 0 in the fourth and following columns. Likewise, the third row of the table of Figure 5.1 shows the four subshrubs (first, second, third, and fourth columns) and the infinite chaotic bands (fifth and following columns) of the shrub of the quaternary hyperbolic component $1 / 1 \cdot 1 / 3 \cdot 1 / 4 \cdot 1 / 2 \cdot 1 / 2[11-13,19]$. Finally, the $(i-1)$ th row of the table of Figure 5.1 shows the shrub of the $i$-ary hyperbolic component $1 / 1 \cdot 1 / 3$. $1 / 4(\cdot 1 / 2)^{i-2}[11-13,19]$ which in the first $i$ columns should show, if the figure had space enough, the $i$ subshrubs, and next the infinite chaotic bands of shrub . 


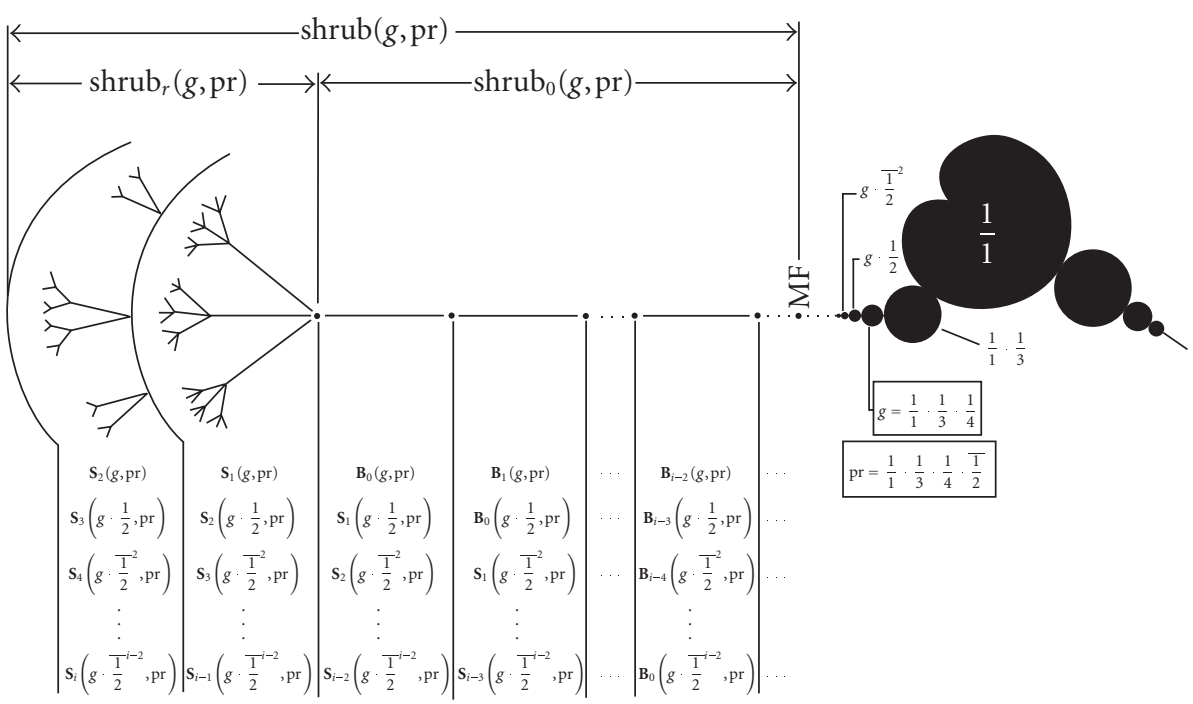

Figure 5.1. Sketch of the secondary route through the secondary hyperbolic component $1 / 1 \cdot 1 / 3$. $1 / 4$, showing the periodic route and the shrub with $\operatorname{shrub}_{0}(1 / 1 \cdot 1 / 3 \cdot 1 / 4,1 / 1 \cdot 1 / 3 \cdot 1 / 4 \cdot \overline{1 / 2})$ and $\operatorname{shrub}_{r}(1 / 1 \cdot 1 / 3 \cdot 1 / 4,1 / 1 \cdot 1 / 3 \cdot 1 / 4 \cdot \overline{1 / 2})$. The lower part shows a table with different names of each chaotic band.

By observing the canonical forms of the table of Figure 5.1 the generators of each one of the chaotic bands of $\operatorname{shrub}_{0}(1 / 1 \cdot 1 / 3 \cdot 1 / 4,1 / 1 \cdot 1 / 3 \cdot 1 / 4 \cdot \overline{1 / 2})$ are seen: $1 / 1 \cdot 1 / 3 \cdot 1 / 4$, $1 / 1 \cdot 1 / 3 \cdot 1 / 4 \cdot 1 / 2,1 / 1 \cdot 1 / 3 \cdot 1 / 4 \cdot 1 / 2 \cdot 1 / 2, \ldots$, that is, the hyperbolic components of the Myrberg-Feigenbaum route. However, the two generators of the two subshrubs of the $\operatorname{shrub} \operatorname{shrub}_{r}(1 / 1 \cdot 1 / 3 \cdot 1 / 4,1 / 1 \cdot 1 / 3 \cdot 1 / 4 \cdot \overline{1 / 2})$ cannot be seen right away because the canonical form is not made explicit in the first two columns of the table. To solve this problem, two new rows are calculated by applying the subshrubs rule and the chaotic bands rule, that will be the first two rows of the new Table 5.1. In this table,

$$
\begin{array}{cc}
\alpha_{1}=\frac{1}{1}, \frac{1}{1} \cdot \frac{1}{3} \cdot \frac{1}{4} \cdot \frac{1}{2}=\frac{1}{1}, \mathrm{pr} ; & \alpha_{2}=\frac{1}{1} \cdot \frac{1}{3}, \frac{1}{1} \cdot \frac{1}{3} \cdot \frac{1}{4} \cdot \frac{1}{2}=\frac{1}{1} \cdot \frac{1}{3}, \mathrm{pr} ; \\
\alpha_{3}=\frac{1}{1} \cdot \frac{1}{3} \cdot \frac{1}{4}, \frac{1}{1} \cdot \frac{1}{3} \cdot \frac{1}{4} \cdot \frac{\overline{1}}{2}=g, \mathrm{pr} ; & \alpha_{4}=\frac{1}{1} \cdot \frac{1}{3} \cdot \frac{1}{4} \cdot \frac{1}{2}, \frac{1}{1} \cdot \frac{1}{3} \cdot \frac{1}{4} \cdot \frac{1}{2}=g \cdot \frac{1}{2}, \mathrm{pr} \\
\alpha_{5}=\frac{1}{1} \cdot \frac{1}{3} \cdot \frac{1}{4}\left(\cdot \frac{1}{2}\right)^{i-2}, \frac{1}{1} \cdot \frac{1}{3} \cdot \frac{1}{4} \cdot \frac{1}{2}=g\left(\cdot \frac{1}{2}\right)^{i-2}, \mathrm{pr} .
\end{array}
$$

The transformation rule is applied in order to have all the data in chaotic bands form. If Table 5.1 is considered a matrix, again the main cardioid is the canonical form of the corresponding chaotic bands, and the successive generators of the chaotic bands are $1 / 1,1 / 1 \cdot 1 / 3,1 / 1 \cdot 1 / 3 \cdot 1 / 4,1 / 1 \cdot 1 / 3 \cdot 1 / 4 \cdot 1 / 2,1 / 1 \cdot 1 / 3 \cdot 1 / 4 \cdot 1 / 2 \cdot 1 / 2, \ldots$, that are the hyperbolic components of the periodic route. $1 / 1$ and $1 / 1 \cdot 1 / 3$ constitute the generation route and are the generators of the two subshrubs of the shrub remainder, and 
Table 5.1

\begin{tabular}{ccccccc}
\hline$B_{0}\left(\alpha_{1}\right)$ & $B_{1}\left(\alpha_{1}\right)$ & $B_{2}\left(\alpha_{1}\right)$ & $B_{3}\left(\alpha_{1}\right)$ & $\ldots$ & $B_{i}\left(\alpha_{1}\right)$ & $\ldots$ \\
$B_{-1}\left(\alpha_{2}\right)$ & $B_{0}\left(\alpha_{2}\right)$ & $B_{1}\left(\alpha_{2}\right)$ & $B_{2}\left(\alpha_{2}\right)$ & $\ldots$ & $B_{i-1}\left(\alpha_{2}\right)$ & $\ldots$ \\
$B_{-2}\left(\alpha_{3}\right)$ & $B_{-1}\left(\alpha_{3}\right)$ & $B_{0}\left(\alpha_{3}\right)$ & $B_{1}\left(\alpha_{3}\right)$ & $\ldots$ & $B_{i-2}\left(\alpha_{3}\right)$ & $\ldots$ \\
$B_{-3}\left(\alpha_{4}\right)$ & $B_{-2}\left(\alpha_{4}\right)$ & $B_{-1}\left(\alpha_{4}\right)$ & $B_{0}\left(\alpha_{4}\right)$ & $\ldots$ & $B_{i-3}\left(\alpha_{4}\right)$ & $\ldots$ \\
$\ldots$ & $\ldots$ & $\ldots$ & $\ldots$ & $\ldots$ & $\ldots$ & $\ldots$ \\
$B_{-i}\left(\alpha_{5}\right)$ & $B_{-i+1}\left(\alpha_{5}\right)$ & $B_{-i+2}\left(\alpha_{5}\right)$ & $B_{-i+3}\left(\alpha_{5}\right)$ & $\ldots$ & $B_{0}\left(\alpha_{5}\right)$ & $\ldots$ \\
$\ldots$ & $\ldots$ & $\ldots$ & $\ldots$ & $\ldots$ & $\ldots$ & $\ldots$ \\
\hline
\end{tabular}

$1 / 1 \cdot 1 / 3 \cdot 1 / 4,1 / 1 \cdot 1 / 3 \cdot 1 / 4 \cdot 1 / 2,1 / 1 \cdot 1 / 3 \cdot 1 / 4 \cdot 1 / 2 \cdot 1 / 2, \ldots$ constitute the MyrbergFeigenbaum route and are the generators of the infinity of chaotic bands of shrub .

Let us see next a tertiary route. We call tertiary route to a route whose generator is a tertiary hyperbolic component $1 / 1 \cdot q_{1} / p_{1} \cdot q_{2} / p_{2} \cdot q_{3} / p_{3}$, as $1 / 1 \cdot 1 / 3 \cdot 1 / 4 \cdot 1 / 5$ to be seen next. The periodic route is $1 / 1,1 / 1 \cdot 1 / 3,1 / 1 \cdot 1 / 3 \cdot 1 / 4$ (generation route) followed by $1 / 1 \cdot 1 / 3 \cdot 1 / 4 \cdot 1 / 5$ and the hyperbolic components of its period doubling cascade (Myrberg-Feigenbaum route). After the Myrberg-Feigenbaum point, the chaotic route, described by shrubs, is reached.

The upper part of Figure 5.2 shows the sketch of both the periodic route and the $\operatorname{shrub}(1 / 1 \cdot 1 / 3 \cdot 1 / 4 \cdot 1 / 5, \mathrm{pr})$, where $\mathrm{pr}=1 / 1 \cdot 1 / 3 \cdot 1 / 4 \cdot 1 / 5 \cdot \overline{1 / 2}$ is the periodic route. The first row of the table of the lower part of Figure 5.2 shows such $\operatorname{shrub}(1 / 1 \cdot 1 / 3$. $1 / 4 \cdot 1 / 5, \mathrm{pr})$. $\operatorname{shrub}_{r}(1 / 1 \cdot 1 / 3 \cdot 1 / 4 \cdot 1 / 5, \mathrm{pr})$ is given in the first three columns: $\mathbf{S}_{3}(1 / 1 \cdot$ $1 / 3 \cdot 1 / 4 \cdot 1 / 5$, pr), $S_{2}\left(1 / 1 \cdot 1 / 3 \cdot 1 / 4 \cdot 1 / 5\right.$, pr), and $S_{1}(1 / 1 \cdot 1 / 3 \cdot 1 / 4 \cdot 1 / 5$, pr). The infinity of chaotic bands of $\operatorname{shrub}_{0}(1 / 1 \cdot 1 / 3 \cdot 1 / 4 \cdot 1 / 5$, pr $)$ are given in the following columns: $\mathbf{B}_{0}(1 / 1 \cdot 1 / 3 \cdot 1 / 4 \cdot 1 / 5, \mathrm{pr}), \mathbf{B}_{1}(1 / 1 \cdot 1 / 3 \cdot 1 / 4 \cdot 1 / 5, \mathrm{pr}), \mathbf{B}_{2}(1 / 1 \cdot 1 / 3 \cdot 1 / 4 \cdot 1 / 5, \mathrm{pr}), \ldots$, $\mathbf{B}_{i}(1 / 1 \cdot 1 / 3 \cdot 1 / 4 \cdot 1 / 5, \mathrm{pr}), \ldots[11-13,19]$. The second row of the table of Figure 5.2 shows the shrub of the first disc of the period doubling cascade (which is the quaternary hyperbolic component $1 / 1 \cdot 1 / 3 \cdot 1 / 4 \cdot 1 / 5 \cdot 1 / 2)$ the $\operatorname{shrub}(1 / 1 \cdot 1 / 3 \cdot 1 / 4 \cdot 1 / 5 \cdot 1 / 2$, pr $)[11-$ $13,19]$. The first four columns show the four subshrubs of the shrub remainder, and

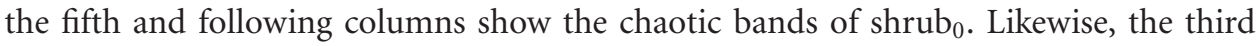
row of the table of Figure 5.2 shows the five subshrubs (first, second, third, fourth, and fifth columns) and the infinity of chaotic bands (sixth and following columns) of the shrub of the 5 -ary hyperbolic component $1 / 1 \cdot 1 / 3 \cdot 1 / 4 \cdot 1 / 5 \cdot 1 / 2 \cdot 1 / 2[11-13,19]$. Finally, the $(i-2)$ th row of Table 6.1 shows the shrub of the $i$-ary hyperbolic component $1 / 1 \cdot 1 / 3 \cdot 1 / 4 \cdot 1 / 5(\cdot 1 / 2)^{i-3}[11-13,19]$. The first $i$ columns show the $i$ subshrubs of the shrub remainder, and the following columns the chaotic bands of shrub ${ }_{0}$ (again, the figure is not complete due to space problems).

The generators of each one of the chaotic bands of $\operatorname{shrub}_{0}(1 / 1 \cdot 1 / 3 \cdot 1 / 4 \cdot 1 / 5$, pr $)$ are $1 / 1 \cdot 1 / 3 \cdot 1 / 4 \cdot 1 / 5,1 / 1 \cdot 1 / 3 \cdot 1 / 4 \cdot 1 / 5 \cdot 1 / 2,1 / 1 \cdot 1 / 3 \cdot 1 / 4 \cdot 1 / 5 \cdot 1 / 2 \cdot 1 / 2, \ldots$, as can be seen from the canonical forms of the table of Figure 5.2; that is to say, the generators are the hyperbolic components of the Myrberg-Feigenbaum route. To obtain the generators of the three subshrubs of $\operatorname{shrub}_{r}(1 / 1 \cdot 1 / 3 \cdot 1 / 4 \cdot 1 / 5$, pr $)$ three new rows have to be 


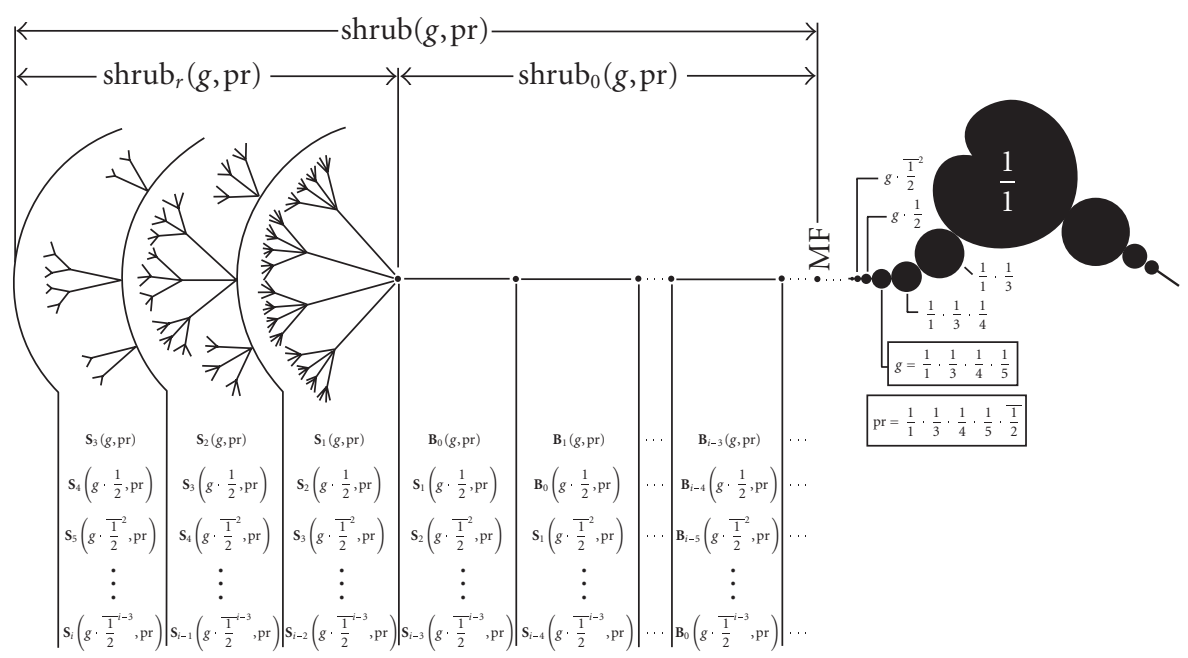

Figure 5.2. Sketch of the tertiary route through the tertiary hyperbolic component $1 / 1 \cdot 1 / 3 \cdot 1 / 4 \cdot 1 / 5$, showing the periodic route and the chaotic route, $\operatorname{shrub}(1 / 1 \cdot 1 / 3 \cdot 1 / 4 \cdot 1 / 5, \mathrm{pr})$. The lower part shows a table with different names of each chaotic band.

Table 5.2

\begin{tabular}{cccccccc}
\hline$B_{0}\left(\beta_{1}\right)$ & $B_{1}\left(\beta_{1}\right)$ & $B_{2}\left(\beta_{1}\right)$ & $B_{3}\left(\beta_{1}\right)$ & $B_{4}\left(\beta_{1}\right)$ & $\ldots$ & $B_{i}\left(\beta_{1}\right)$ & $\ldots$ \\
$B_{-1}\left(\beta_{2}\right)$ & $B_{0}\left(\beta_{2}\right)$ & $B_{1}\left(\beta_{2}\right)$ & $B_{2}\left(\beta_{2}\right)$ & $B_{3}\left(\beta_{2}\right)$ & $\ldots$ & $B_{i-1}\left(\beta_{2}\right)$ & $\ldots$ \\
$B_{-2}\left(\beta_{3}\right)$ & $B_{-1}\left(\beta_{3}\right)$ & $B_{0}\left(\beta_{3}\right)$ & $B_{1}\left(\beta_{3}\right)$ & $B_{2}\left(\beta_{3}\right)$ & $\ldots$ & $B_{i-2}\left(\beta_{3}\right)$ & $\ldots$ \\
$B_{-3}\left(\beta_{4}\right)$ & $B_{-2}\left(\beta_{4}\right)$ & $B_{-1}\left(\beta_{4}\right)$ & $B_{0}\left(\beta_{4}\right)$ & $B_{1}\left(\beta_{4}\right)$ & $\ldots$ & $B_{i-3}\left(\beta_{4}\right)$ & $\ldots$ \\
$B_{-4}\left(\beta_{5}\right)$ & $B_{-3}\left(\beta_{5}\right)$ & $B_{-2}\left(\beta_{5}\right)$ & $B_{-1}\left(\beta_{5}\right)$ & $B_{0}\left(\beta_{5}\right)$ & $\ldots$ & $B_{i-4}\left(\beta_{5}\right)$ & $\ldots$ \\
$\ldots$ & $\ldots$ & $\ldots$ & $\ldots$ & $\ldots$ & $\ldots$ & $\ldots$ & $\ldots$ \\
$B_{-i}\left(\beta_{6}\right)$ & $B_{-i+1}\left(\beta_{6}\right)$ & $B_{-i+2}\left(\beta_{6}\right)$ & $B_{-i+3}\left(\beta_{6}\right)$ & $B_{-i+4}\left(\beta_{6}\right)$ & $\ldots$ & $B_{0}\left(\beta_{6}\right)$ & $\ldots$ \\
$\ldots$ & $\ldots$ & $\ldots$ & $\ldots$ & $\ldots$ & $\ldots$ & $\ldots$ & $\ldots$ \\
\hline
\end{tabular}

calculated, which will be the first three rows of the new Table 5.2. In this table,

$$
\begin{gathered}
\beta_{1}=\frac{1}{1}, \mathrm{pr} ; \quad \beta_{2}=\frac{1}{1} \cdot \frac{1}{3}, \mathrm{pr} ; \quad \beta_{3}=\frac{1}{1} \cdot \frac{1}{3} \cdot \frac{1}{4}, \mathrm{pr} ; \\
\beta_{4}=\frac{1}{1} \cdot \frac{1}{3} \cdot \frac{1}{4} \cdot \frac{1}{5}, \mathrm{pr}=g, \mathrm{pr} ; \quad \beta_{5}=\frac{1}{1} \cdot \frac{1}{3} \cdot \frac{1}{4} \cdot \frac{1}{5} \cdot \frac{1}{2}, \mathrm{pr}=g \cdot \frac{1}{2}, \mathrm{pr} \\
\beta_{6}=\frac{1}{1} \cdot \frac{1}{3} \cdot \frac{1}{4} \cdot \frac{1}{5}\left(\cdot \frac{1}{2}\right)^{i-3}, \mathrm{pr}=g\left(\cdot \frac{1}{2}\right)^{i-3}, \mathrm{pr} .
\end{gathered}
$$

Transformation $\mathbf{S}_{i}=\mathbf{B}_{-i}$ has been applied again in order to have all the expressions as chaotic bands. The main diagonal of Table 5.2 is the canonical form; therefore, the successive generators of the chaotic bands are $1 / 1,1 / 1 \cdot 1 / 3,1 / 1 \cdot 1 / 3 \cdot 1 / 4,1 / 1 \cdot 1 / 3 \cdot 1 / 4 \cdot 1 / 5$, $1 / 1 \cdot 1 / 3 \cdot 1 / 4 \cdot 1 / 5 \cdot 1 / 2,1 / 1 \cdot 1 / 3 \cdot 1 / 4 \cdot 1 / 5 \cdot 1 / 2 \cdot 1 / 2, \ldots$, that again are the hyperbolic components of the periodic route. 


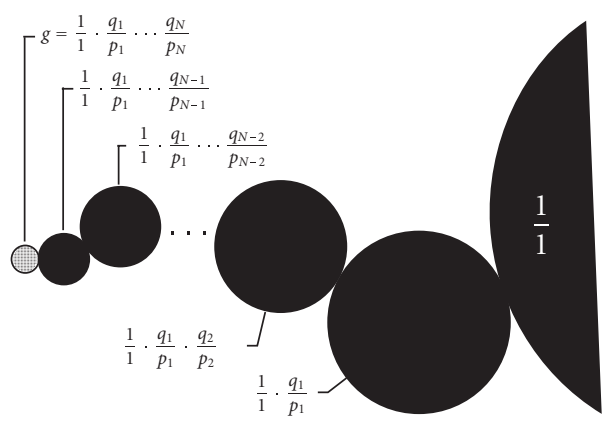

(a) Generation route

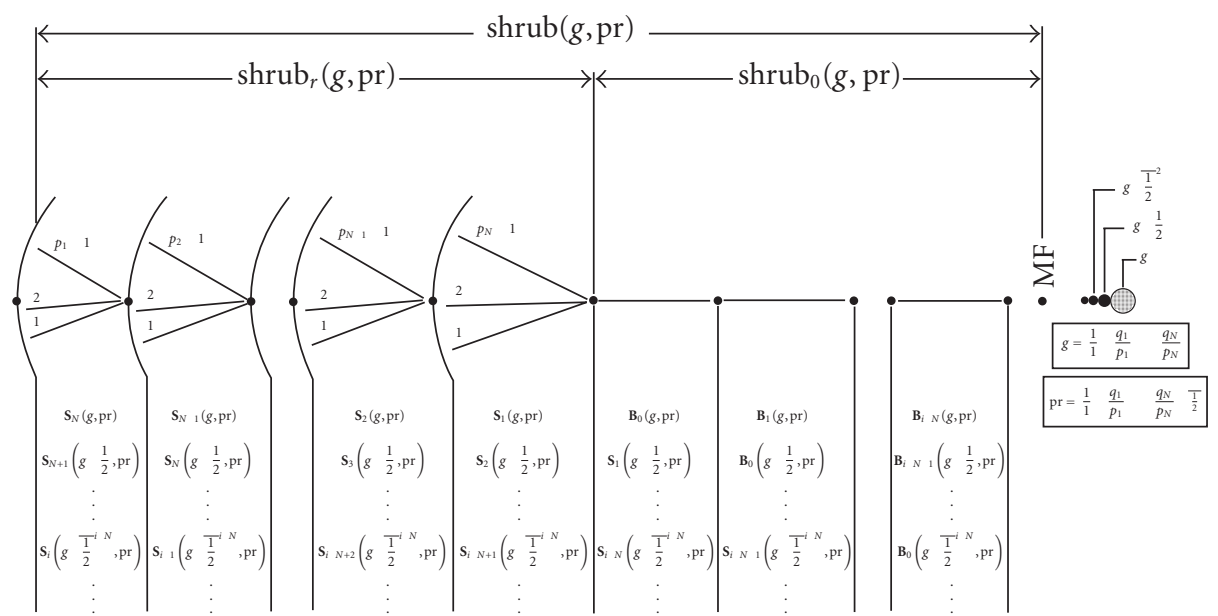

(b) MF route and chaotic route, this last one described by $\operatorname{shrub}\left(1 / 1 \cdot q_{1} / p_{1} \cdots q_{N} / p_{N}\right.$, pr). The lower part shows a table with different names of each chaotic band

Figure 6.1. Sketch of the $N$-ary route through the $N$-ary hyperbolic component $1 / 1 \cdot q_{1} / p_{1} \cdots q_{N} / p_{N}$.

\section{6. $N$-ary route}

Since all the routes analyzed have a similar behaviour, the general case can be given. The route whose generator is the $N$-ary hyperbolic component $1 / 1 \cdot q_{1} / p_{1} \cdots q_{N} / p_{N}$ is called $N$-ary route. As always, the periodic route has two parts. The first part is $1 / 1,1 / 1 \cdot q_{1} / p_{1}$, $1 / 1 \cdot q_{1} / p_{1} \cdot q_{2} / p_{2}, \ldots, 1 / 1 \cdot q_{1} / p_{1} \cdots q_{N-1} / p_{N-1}$, that constitutes the generation route (see Figure 6.1(a)) and the second part is $1 / 1 \cdot q_{1} / p_{1} \cdots q_{N} / p_{N}, 1 / 1 \cdot q_{1} / p_{1} \cdots q_{N} / p_{N}$. $1 / 2,1 / 1 \cdot q_{1} / p_{1} \cdots q_{N} / p_{N} \cdot 1 / 2 \cdot 1 / 2, \ldots$, that constitutes the Myrberg-Feigenbaum route (see Figure 6.1(b)). After the Myrberg-Feigenbaum route, the chaotic route (described by the shrubs) is reached.

On the left of the Myrberg-Feigenbaum point, the upper part of Figure 6.1(b) shows the general sketch of $\operatorname{shrub}\left(1 / 1 \cdot q_{1} / p_{1} \cdots q_{N} / p_{N}, \mathrm{pr}\right)$, where $\mathrm{pr}=1 / 1 \cdot q_{1} / p_{1} \cdots q_{N} / p_{N} \cdot$ 
$\overline{1 / 2}$ is the periodic route. $\operatorname{shrub}\left(1 / 1 \cdot q_{1} / p_{1} \cdots q_{N} / p_{N}, \mathrm{pr}\right)$ has also two parts, $\operatorname{shrub}_{0}(1 / 1 \cdot$ $q_{1} / p_{1} \cdots q_{N} / p_{N}$, pr $)$ and $\operatorname{shrub}_{r}\left(1 / 1 \cdot q_{1} / p_{1} \cdots q_{N} / p_{N}\right.$, pr $) . \operatorname{shrub}_{0}\left(1 / 1 \cdot q_{1} / p_{1} \cdots q_{N} / p_{N}\right.$, pr) is constituted by the infinity of chaotic bands of the hyperbolic component $1 / 1$. $q_{1} / p_{1} \cdots q_{N} / p_{N}$, and $\operatorname{shrub}_{r}\left(1 / 1 \cdot q_{1} / p_{1} \cdots q_{N} / p_{N}\right.$, pr $)$ is constituted by $N$ subshrubs, $S_{1}\left(1 / 1 \cdot q_{1} / p_{1} \cdots q_{N} / p_{N}, p r\right), S_{2}\left(1 / 1 \cdot q_{1} / p_{1} \cdots q_{N} / p_{N}, p r\right), \ldots, S_{N-1}\left(1 / 1 \cdot q_{1} / p_{1} \cdots q_{N} /\right.$ $p_{N}$, pr) and $S_{N}\left(1 / 1 \cdot q_{1} / p_{1} \cdots q_{N} / p_{N}\right.$, pr $)$, because $1 / 1 \cdot q_{1} / p_{1} \cdots q_{N} / p_{N}$ is an $N$-ary hyperbolic component. The first row of the table of the lower part of Figure 6.1(b) shows $\operatorname{shrub}\left(1 / 1 \cdot q_{1} / p_{1} \cdots q_{N} / p_{N}, \mathrm{pr}\right) . \operatorname{shrub}_{r}\left(1 / 1 \cdot q_{1} / p_{1} \cdots q_{N} / p_{N}, \mathrm{pr}\right)$ is given in the first $N$ columns: $S_{N}\left(1 / 1 \cdot q_{1} / p_{1} \cdots q_{N} / p_{N}, \mathrm{pr}\right), \mathbf{S}_{N-1}\left(1 / 1 \cdot q_{1} / p_{1} \cdots q_{N} / p_{N}, \mathrm{pr}\right), \ldots, \mathbf{S}_{2}\left(1 / 1 \cdot q_{1} /\right.$ $\left.p_{1} \cdots q_{N} / p_{N}, \mathrm{pr}\right)$, and $\boldsymbol{S}_{1}\left(1 / 1 \cdot q_{1} / p_{1} \cdots q_{N} / p_{N}, \mathrm{pr}\right)$. $\operatorname{shrub}_{0}\left(1 / 1 \cdot q_{1} / p_{1} \cdots q_{N} / p_{N}, \mathrm{pr}\right)$ is given in the following columns: $\mathbf{B}_{0}\left(1 / 1 \cdot q_{1} / p_{1} \cdots q_{N} / p_{N}, \mathrm{pr}\right), \mathbf{B}_{1}\left(1 / 1 \cdot q_{1} / p_{1} \cdots q_{N} / p_{N}\right.$, pr), $\mathbf{B}_{2}\left(1 / 1 \cdot q_{1} / p_{1} \cdots q_{N} / p_{N}, \operatorname{pr}\right), \ldots, \mathbf{B}_{i}\left(1 / 1 \cdot q_{1} / p_{1} \cdots q_{N} / p_{N}, p r\right), \ldots[11-13,19]$.

The second row of the table of Figure 6.1(b) shows the shrub(1/1 $\cdot q_{1} / p_{1} \cdots q_{N} / p_{N} \cdot$ $1 / 2$,pr) $[11-13,19]$, which is the shrub of the first disc of the period doubling cascade, the $(N+1)$-ary hyperbolic component $1 / 1 \cdot q_{1} / p_{1} \cdots q_{N} / p_{N} \cdot 1 / 2$. The $N+1$ subshrubs of the shrub remainder are shown in the first $N+1$ columns, and the infinity of chaotic bands of shrub 0 are shown in the $(N+2)$ th and following columns. Likewise, the third row of the table of Figure $6.1(\mathrm{~b}) \operatorname{shows} \operatorname{shrub}\left(1 / 1 \cdot q_{1} / p_{1} \cdots q_{N} / p_{N} \cdot 1 / 2 \cdot\right.$ $1 / 2, \mathrm{pr})$, which is the shrub of the second disc of the period doubling cascade, the $(N+2)$ ary hyperbolic component $1 / 1 \cdot q_{1} / p_{1} \cdots q_{N} / p_{N} \cdot 1 / 2 \cdot 1 / 2[11-13,19]$. The first $N+2$ columns show the $N+2$ subshrubs of the shrub remainder, and the $(N+3)$ th and follow-

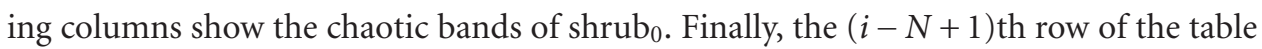
of Figure 6.1(b) shows $\operatorname{shrub}\left(1 / 1 \cdot q_{1} / p_{1} \cdots q_{N} / p_{N}(\cdot 1 / 2)^{i-N}\right.$, pr $)$, which is the shrub of the $(i-N)$ th disc of the period doubling cascade, the $i$-ary hyperbolic component $1 / 1$. $q_{1} / p_{1} \cdots q_{N} / p_{N}(\cdot 1 / 2)^{i-N}[11-13,19] . \operatorname{shrub}\left(1 / 1 \cdot q_{1} / p_{1} \cdots q_{N} / p_{N}(\cdot 1 / 2)^{i-N}\right.$, pr $)$ has the $i$ subshrubs of shrub ${ }_{r}$ in the first $i$ columns, and the successive chaotic bands of $\operatorname{shrub}_{0}$ in the following columns.

From the canonical forms of the table of Figure 6.1(b), the generators of the chaotic bands of $\operatorname{shrub}_{0}\left(1 / 1 \cdot q_{1} / p_{1} \cdots q_{N} / p_{N}\right.$, pr $)$ are $1 / 1 \cdot q_{1} / p_{1} \cdots q_{N} / p_{N}, 1 / 1 \cdot q_{1} / p_{1} \cdots q_{N} /$ $p_{N} \cdot 1 / 2,1 / 1 \cdot q_{1} / p_{1} \cdots q_{N} / p_{N} \cdot 1 / 2 \cdot 1 / 2, \ldots$, that are the hyperbolic components of the Myrberg-Feigenbaum route. To obtain the generators of the $N$ subshrubs of $\operatorname{shrub}_{r}(1 / 1$. $\left.q_{1} / p_{1} \cdots q_{N} / p_{N}, p r\right)$, the new $N$ rows are calculated as always, and they will be the first $N$ rows of the new Table 6.1. In this table,

$$
\begin{gathered}
\gamma_{1}=\frac{1}{1}, \mathrm{pr} ; \quad \gamma_{2}=\frac{1}{1} \cdot \frac{q_{1}}{p_{1}}, \mathrm{pr} ; \quad \gamma_{3}=\frac{1}{1} \cdot \frac{q_{1}}{p_{1}} \cdots \frac{q_{N-1}}{p_{N-1}}, \mathrm{pr} ; \\
\gamma_{4}=\frac{1}{1} \cdot \frac{q_{1}}{p_{1}} \cdots \frac{q_{N}}{p_{N}}, \mathrm{pr}=g, \mathrm{pr} ; \quad \gamma_{5}=\frac{1}{1} \cdot \frac{q_{1}}{p_{1}} \cdots \frac{q_{N}}{p_{N}} \cdot \frac{1}{2}, \mathrm{pr}=g \cdot \frac{1}{2}, \mathrm{pr} ; \\
\gamma_{6}=\frac{1}{1} \cdot \frac{q_{1}}{p_{1}} \cdots \frac{q_{N}}{p_{N}}\left(\cdot \frac{1}{2}\right)^{i-N}, \mathrm{pr}=g\left(\cdot \frac{1}{2}\right)^{i-N}, \mathrm{pr} .
\end{gathered}
$$

The transformation $S_{i}=B_{-i}$ is again applied in order to have all the expressions as chaotic bands. The main diagonal of Table 6.1 is the canonical form, therefore the generators of 
Table 6.1

\begin{tabular}{|c|c|c|c|c|c|c|c|}
\hline$B_{0}\left(\gamma_{1}\right)$ & $B_{1}\left(\gamma_{1}\right)$ & $\cdots$ & $B_{N-1}\left(\gamma_{1}\right)$ & $B_{N}\left(\gamma_{1}\right)$ & $B_{N+1}\left(\gamma_{1}\right)$ & $B_{i}\left(\gamma_{1}\right)$ & \\
\hline$B_{-1}\left(\gamma_{2}\right)$ & $B_{0}\left(\gamma_{2}\right)$ & $\cdots$ & $B_{N-2}\left(\gamma_{2}\right)$ & $B_{N-1}\left(\gamma_{2}\right)$ & $B_{N}\left(\gamma_{2}\right)$ & $\cdots \quad B_{i-1}\left(\gamma_{2}\right)$ & $\ldots$ \\
\hline$\ldots$ & $\ldots$ & $\cdots$ & 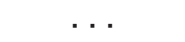 & $\ldots$ & . & $\ldots$ & $\ldots$ \\
\hline$B_{-N+1}\left(\gamma_{3}\right)$ & $B_{-N+2}\left(\gamma_{3}\right)$ & & $B_{0}\left(\gamma_{3}\right)$ & $B_{1}\left(\gamma_{3}\right)$ & $B_{2}\left(\gamma_{3}\right)$ & $\cdots B_{i-N+1}\left(\gamma_{3}\right)$ & \\
\hline$B_{-N}\left(\gamma_{4}\right)$ & $B_{-N+1}\left(\gamma_{4}\right)$ & $\cdots$ & $B_{-1}\left(\gamma_{4}\right)$ & $B_{0}\left(\gamma_{4}\right)$ & $B_{1}\left(\gamma_{4}\right)$ & $\cdots \quad B_{i-N}\left(\gamma_{4}\right)$ & . \\
\hline$B_{-N-1}\left(\gamma_{5}\right)$ & $B_{-N}\left(\gamma_{5}\right)$ & $\cdots$ & $B_{-2}\left(\gamma_{5}\right)$ & $B_{-1}\left(\gamma_{5}\right)$ & $B_{0}\left(\gamma_{5}\right)$ & $\cdots B_{i-N-1}\left(\gamma_{5}\right)$ & 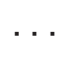 \\
\hline$\cdots$ & $\cdots$ & $\cdots$ & $\cdots$ & . & $\cdots$ & $\cdots$ & $\cdots$ \\
\hline$B_{-i}\left(\gamma_{6}\right)$ & $B_{-i+1}\left(\gamma_{6}\right)$ & $\cdots$ & $B_{-i+N-1}\left(\gamma_{6}\right)$ & $B_{-i+N}\left(\gamma_{6}\right)$ & $B_{-i+N+1}\left(\gamma_{6}\right)$ & $B_{0}\left(\gamma_{6}\right)$ & \\
\hline$\ldots$ & $\cdots$ & $\cdots$ & $\cdots$ & $\cdots$ & $\cdots$ & $\cdots$ & $\cdots$ \\
\hline
\end{tabular}

the chaotic bands are $1 / 1,1 / 1 \cdot q_{1} / p_{1}, 1 / 1 \cdot q_{1} / p_{1} \cdot q_{2} / p_{2}, \ldots, 1 / 1 \cdot q_{1} / p_{1} \cdots q_{N-1} / p_{N-1}$, $1 / 1 \cdot q_{1} / p_{1} \cdots q_{N} / p_{N}, 1 / 1 \cdot q_{1} / p_{1} \cdots q_{N} / p_{N} \cdot 1 / 2,1 / 1 \cdot q_{1} / p_{1} \cdots q_{N} / p_{N} \cdot 1 / 2 \cdot 1 / 2, \ldots$, that are again the hyperbolic components of the periodic route. We already saw that the generators of the chaotic bands of shrub 0 are the hyperbolic components of the MyrbergFeigenbaum route: $1 / 1 \cdot q_{1} / p_{1} \cdots q_{N} / p_{N}, 1 / 1 \cdot q_{1} / p_{1} \cdots q_{N} / p_{N} \cdot 1 / 2,1 / 1 \cdot q_{1} / p_{1} \cdots q_{N} /$ $p_{N} \cdot 1 / 2 \cdot 1 / 2, \ldots$. But in addition, the generators of the $N$ subshrubs of shrub ${ }_{r}$ are the $N$ hyperbolic components of the generation route: $1 / 1,1 / 1 \cdot q_{1} / p_{1}, 1 / 1 \cdot q_{1} / p_{1} \cdot q_{2} / p_{2}, \ldots$, $1 / 1 \cdot q_{1} / p_{1} \cdots q_{N-1} / p_{N-1}$.

Therefore, the general case of an $N$-ary route has a similar behaviour to the one dimensional case with only one route. Hence, what was seen in the one-dimensional case, that has been used in our inductive process until the $N$-ary case, can be generalized. Thus, we can say now in general that subshrubs have the same nature as chaotic bands. Since they have the same nature, they are equivalent, and we can state that the transformation rule between subshrubs and chaotic bands, (3.1): $\mathbf{S}_{i}(\mathrm{hc}, \mathrm{pr})=\mathbf{B}_{-i}(\mathrm{hc}, \mathrm{pr})$, has a general character. From this equation, it is evident that $\mathbf{S}_{0}(\mathrm{hc}, \mathrm{pr})=\mathbf{B}_{0}(\mathrm{hc}, \mathrm{pr})$, which is the canonical form to call a chaotic band or a subshrub among all the possible ways. In the canonical form $\mathrm{hc}=$ generator; that is to say, the hyperbolic component of the canonical form is the generator of the corresponding chaotic band.

Likewise, we have in general the rule for chaotic bands, that shows the different ways to call the same chaotic band, and can be expressed as

$$
\begin{aligned}
\mathbf{B}_{i}\left(\frac{1}{1} \cdot \frac{q_{1}}{p_{1}} \cdots \frac{q_{j}}{p_{j}}, \mathrm{pr}\right) & =\mathbf{B}_{i+1}\left(\frac{1}{1} \cdot \frac{q_{1}}{p_{1}} \cdots \frac{q_{j-1}}{p_{j-1}}, \mathrm{pr}\right) \\
& =\mathbf{B}_{i-1}\left(\frac{1}{1} \cdot \frac{q_{1}}{p_{1}} \cdots \frac{q_{j+1}}{p_{j+1}}, \mathrm{pr}\right)
\end{aligned}
$$

that is to say, if the index increases one unit, then the hyperbolic component decreases one factor (it becomes the preceding hyperbolic component of the periodic route), and if the index decreases one unit, then the hyperbolic component increases one factor (it becomes the following hyperbolic component of the periodic route). 
The same general character can be considered in the case of the rule for subshrubs, that shows the different ways to call the same subshrub:

$$
\mathbf{S}_{i}\left(\frac{1}{1} \cdot \frac{q_{1}}{p_{1}} \cdots \frac{q_{j}}{p_{j}}, \mathrm{pr}\right)=\mathbf{S}_{i+1}\left(\frac{1}{1} \cdot \frac{q_{1}}{p_{1}} \cdots \frac{q_{j+1}}{p_{j+1}}, \mathrm{pr}\right)=\mathbf{S}_{i-1}\left(\frac{1}{1} \cdot \frac{q_{1}}{p_{1}} \cdots \frac{q_{j-1}}{p_{j-1}}, \mathrm{pr}\right)
$$

that is to say, if the index increases one unit, then the hyperbolic component also increases one factor (it becomes the following hyperbolic component of the periodic route), and if the index decreases one unit, then the hyperbolic component also decreases one factor (it becomes the preceding hyperbolic component of the periodic route).

In accordance with what has just been seen, the generalization of the shrub can be given. As is known, shrubs have two parts: $\operatorname{shrub}_{0}$ and $\operatorname{shrub}_{r} \cdot \operatorname{shrub}_{0}\left(1 / 1 \cdot q_{1} / p_{1} \cdots q_{N} /\right.$ $\left.p_{N}, \mathrm{pr}\right)$ is constituted by the infinity of chaotic bands of the $N$-ary hyperbolic component $1 / 1 \cdot q_{1} / p_{1} \cdots q_{N} / p_{N}$, that is,

$$
\operatorname{shrub}_{0}\left(\frac{1}{1} \cdot \frac{q_{1}}{p_{1}} \cdots \frac{q_{N}}{p_{N}}, \mathrm{pr}\right)=\bigcup_{i=0}^{\infty} \mathbf{B}_{i}\left(\frac{1}{1} \cdot \frac{q_{1}}{p_{1}} \cdots \frac{q_{N}}{p_{N}}, \mathrm{pr}\right)=\bigcup_{i=-\infty}^{0} \mathbf{S}_{i}\left(\frac{1}{1} \cdot \frac{q_{1}}{p_{1}} \cdots \frac{q_{N}}{p_{N}}, \mathrm{pr}\right) .
$$

On the other hand, the $\operatorname{shrub}_{r}\left(1 / 1 \cdot q_{1} / p_{1} \cdots q_{N} / p_{N}, \mathrm{pr}\right)$ is constituted by $N$ subshrubs, that is to say

$$
\operatorname{shrub}_{r}\left(\frac{1}{1} \cdot \frac{q_{1}}{p_{1}} \cdots \frac{q_{N}}{p_{N}}, \mathrm{pr}\right)=\bigcup_{i=0}^{N} \mathbf{S}_{i}\left(\frac{1}{1} \cdot \frac{q_{1}}{p_{1}} \cdots \frac{q_{N}}{p_{N}}, \mathrm{pr}\right)=\bigcup_{i=-N}^{0} \mathbf{B}_{i}\left(\frac{1}{1} \cdot \frac{q_{1}}{p_{1}} \cdots \frac{q_{N}}{p_{N}}, \mathrm{pr}\right) .
$$

As can be seen, both the shrub 0 of (6.4) and the shrub ${ }_{r}$ of (6.5) have been expressed in the two possible ways, as chaotic bands and as shrubs. Therefore, the general way of expressing a shrub as chaotic bands is

$$
\operatorname{shrub}\left(\frac{1}{1} \cdot \frac{q_{1}}{p_{1}} \cdots \frac{q_{N}}{p_{N}}, \mathrm{pr}\right)=\bigcup_{i=-N}^{\infty} \mathbf{B}_{i}\left(\frac{1}{1} \cdot \frac{q_{1}}{p_{1}} \cdots \frac{q_{N}}{p_{N}}, \mathrm{pr}\right) .
$$

However, a shrub can also be expressed in a general way as subshrubs as follows:

$$
\operatorname{shrub}\left(\frac{1}{1} \cdot \frac{q_{1}}{p_{1}} \cdots \frac{q_{N}}{p_{N}}, \mathrm{pr}\right)=\bigcup_{i=-\infty}^{N} \mathrm{~S}_{i}\left(\frac{1}{1} \cdot \frac{q_{1}}{p_{1}} \cdots \frac{q_{N}}{p_{N}}, \mathrm{pr}\right) .
$$

Equations (6.6) and (6.7) are the more compact way of expressing a shrub. Nevertheless, if negative indexes are not wanted, the habitual form must be given where both subshrubs and chaotic bands are present:

$$
\operatorname{shrub}\left(\frac{1}{1} \cdot \frac{q_{1}}{p_{1}} \cdots \frac{q_{N}}{p_{N}}, \mathrm{pr}\right)=\bigcup_{i=1}^{N} \mathbf{S}_{i}\left(\frac{1}{1} \cdot \frac{q_{1}}{p_{1}} \cdots \frac{q_{N}}{p_{N}}, \mathrm{pr}\right) \bigcup_{i=0}^{\infty} \mathbf{B}_{i}\left(\frac{1}{1} \cdot \frac{q_{1}}{p_{1}} \cdots \frac{q_{N}}{p_{N}}, \mathrm{pr}\right) .
$$




\section{Conclusions}

This paper clearly shows the equivalence between the two zones of the chaotic region of the Mandelbrot set that always had been thought as different: chaotic bands of shrub ${ }_{0}$ and subshrubs of shrub remainder. This equivalence makes unnecessary the shrub division in shrub $b_{0}$ and shrub remainder, although sometimes we deal with these two parts for convenience.

By basing on the one-dimensional case study that has been already accomplished by us in previous papers, we carry out an analysis of the periodic and chaotic routes whose generators are primary, secondary, ... hyperbolic components, and the generalization for $N$-ary hyperbolic components.

Both a specific subshrub and a specific chaotic band can be called in different ways because they may come from different hyperbolic components. Therefore new rules are needed to go from a name to another, that is, what we do with the rules for subshrubs and the rules for chaotic bands. Likewise, an important conclusion is reached: both subshrubs and chaotic bands have the same nature. Hence, we need rules to go from subshrubs to chaotic bands and vice versa, that is, what we solved by the introduction of the transformation rule.

Among all the names of a subshrub or of a chaotic band, we show what is the most important and we call it canonical form. This canonical form is associated to the generator of the chaotic band.

\section{Acknowledgment}

This work was supported by "Ministerio de Educación y Ciencia" of Spain, Research Grant SEG2004-02418.

\section{References}

[1] B. Branner, The Mandelbrot set, Chaos and Fractals (Providence, RI, 1988), Proc. Sympos. Appl. Math., vol. 39, American Mathematical Society, Rhode Island, 1989, pp. 75-105.

[2] R. L. Devaney, The fractal geometry of the Mandelbrot set. 2. How to count and how to add, Fractals 3 (1995), no. 4, 629-640.

[3] A. Douady and J. H. Hubbard, Étude Dynamique des Polynômes Complexes. Partie I, Publications Mathématiques d'Orsay, vol. 84, Université de Paris-Sud, Département de Mathématiques, Orsay, 1984.

[4] _ Étude Dynamique des Polynômes Complexes. Partie II, Publications Mathématiques d'Orsay, vol. 85, Université de Paris-Sud, Département de Mathématiques, Orsay, 1985.

[5] B. B. Mandelbrot, Fractal aspects of the iteration of $z=l z(1-z)$ for complex $l$ and $z$, Nonlinear Dynamics (R. H. G. Helleman, ed.), Annals of the New York Academy of Sciences, vol. 357, Academy of Sciences, New York, 1980, pp. 249-259.

[6] _ On the quadratic mapping $z \rightarrow z^{2}-\mu$ for complex $\mu$ and $z$ : the fractal structure of its $M$ set, and scaling, Physica D 7 (1983), no. 1-3, 224-239.

[7] N. Metropolis, M. L. Stein, and P. R. Stein, On finite limit sets for transformations on the unit interval, Journal of Combinatorial Theory 15 (1973), no. 1, 25-44. 
[8] J. Milnor, Self-similarity and hairiness in the Mandelbrot set, Computers in Geometry and Topology (Chicago, IL, 1986), Lecture Notes in Pure and Applied Mathematics, vol. 114, Dekker, New York, 1989, pp. 211-257.

[9] J. Milnor and W. Thurston, On iterated maps of the interval, Dynamical Systems (College Park, MD, 1986-1987) (J. Alexander, ed.), Lecture Notes in Math., vol. 1342, Springer, Berlin, 1988, pp. 465-563.

[10] G. Pastor, M. Romera, G. Álvarez, and F. Montoya, Operating with external arguments in the Mandelbrot set antenna, Physica D 171 (2002), no. 1-2, 52-71.

[11] _ Chaotic bands in the Mandelbrot set, Compers \& Graphics 28 (2004), no. 5, 779-784.

[12] _ External arguments for the chaotic bands calculation in the Mandelbrot set, Physica A 353 (2005), 145-158.

[13] On periodic and chaotic regions in the Mandelbrot set, to appear in Chaos, Solitons \& Fractals.

[14] G. Pastor, M. Romera, and F. Montoya, An approach to the ordering of one-dimensional quadratic maps, Chaos, Solitons \& Fractals 7 (1996), no. 4, 565-584.

[15] _ Harmonic structure of one-dimensional quadratic maps, Physical Review E 56 (1997), no. 2, 1476-1483.

[16] G. Pastor, M. Romera, J. C. Sanz-Martín, and F. Montoya, Symbolic sequences of one-dimensional quadratic map points, Physica A 256 (1998), no. 3-4, 369-382.

[17] H.-O. Peitgen and P. H. Richter, The Beauty of Fractals, Springer, Berlin, 1986.

[18] M. Romera, G. Pastor, G. Álvarez, and F. Montoya, Heredity in one-dimensional quadratic maps, Phisical Review E 58 (1998), no. 6, 7214-7218.

[19] Shrubs in the Mandelbrot set ordering, International Journal of Bifurcation and Chaos 13 (2003), no. 8, 2279-2300.

[20] M. Schroeder, Fractals, Chaos, Power Laws, W. H. Freeman, New York, 1991.

[21] A. N. Sharkovsky, Coexistence of cycles of a continuous map of the line into itself, Ukrainian Mathematical Journal 16 (1964), 61-74.

[22] A. N. Sharkovsky, Yu. L. Maĭstrenko, and E. Yu. Romanenko, Difference Equations and Their Applications, Mathematics and Its Applications, vol. 250, Kluwer Academic, Dordrecht, 1993.

[23] W.-M. Zheng and B.-L. Hao, Applied symbolic dynamics, Experimental Study and Characterization of Chaos (B.-L. Hao, ed.), World Scientific, Singapore, 1990, pp. 364-459.

G. Pastor: Departamento de Tratamiento de la Informacĩn y Codificacĩn, Instituto de Física Aplicada, Consejo Superior de Investigaciones Científicas, Serrano 144, 28006 Madrid, Spain

E-mail address: gerardo@iec.csic.es

M. Romera: Departamento de Tratamiento de la Informacín y Codificacin, Instituto de Física Aplicada, Consejo Superior de Investigaciones Científicas, Serrano 144, 28006 Madrid, Spain E-mail address: miguel@iec.csic.es

G. Alvarez: Departamento de Tratamiento de la Informacin y Codificacin, Instituto de Física Aplicada, Consejo Superior de Investigaciones Científicas, Serrano 144, 28006 Madrid, Spain E-mail address: gonzalo@iec.csic.es

D. Arroyo: Departamento de Tratamiento de la Informacin y Codificacin, Instituto de Física Aplicada, Consejo Superior de Investigaciones Científicas, Serrano 144, 28006 Madrid, Spain E-mail address: david.arroyo@iec.csic.es

F. Montoya: Departamento de Tratamiento de la Informacín y Codificacin, Instituto de Física Aplicada, Consejo Superior de Investigaciones Científicas, Serrano 144, 28006 Madrid, Spain E-mail address: fausto@iec.csic.es 


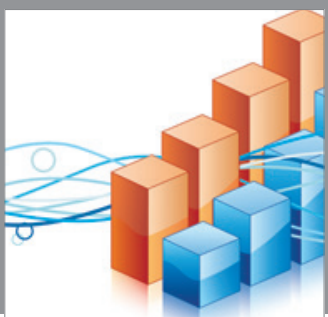

Advances in

Operations Research

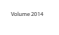

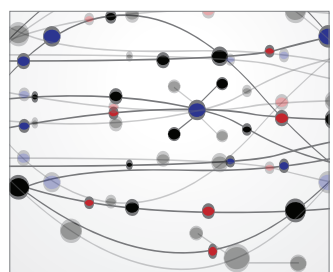

\section{The Scientific} World Journal
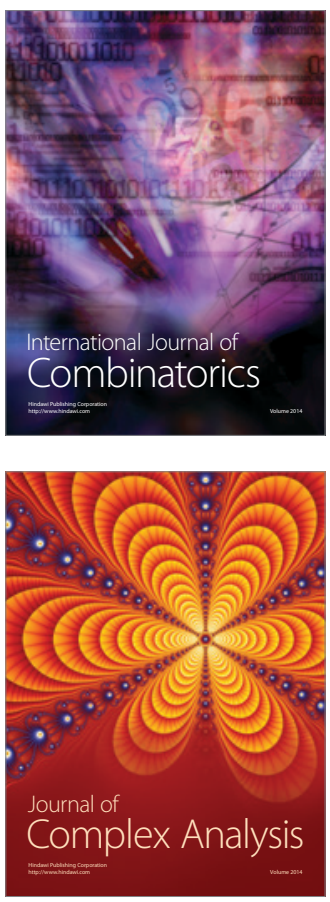

International Journal of

Mathematics and

Mathematical

Sciences
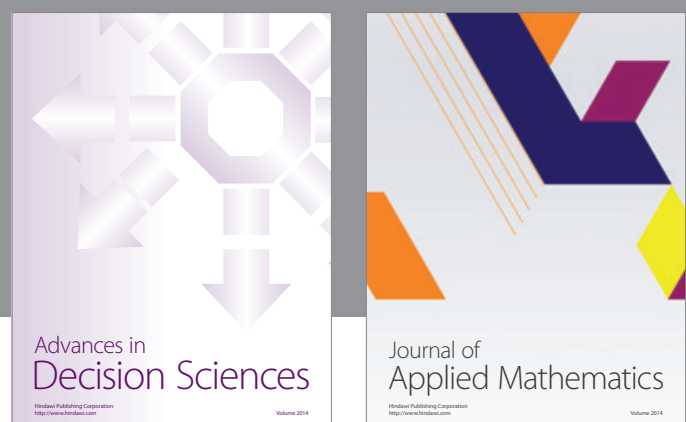

Journal of

Applied Mathematics
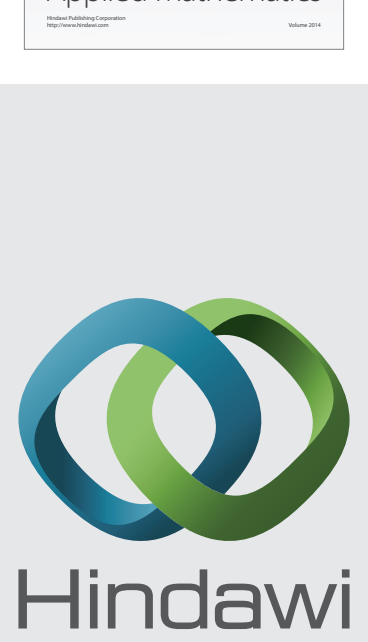

Submit your manuscripts at http://www.hindawi.com
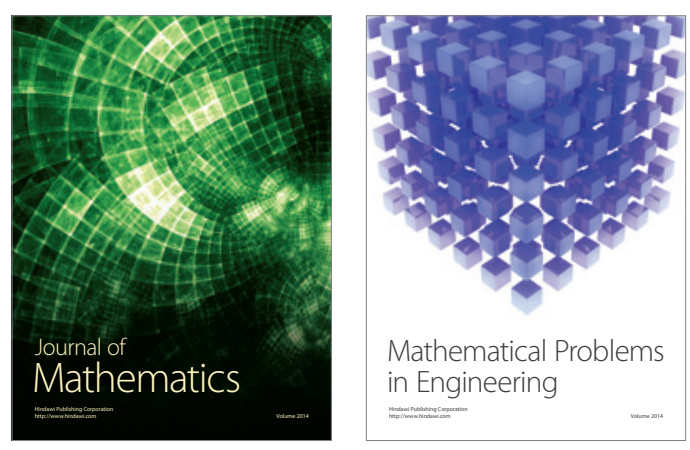

Mathematical Problems in Engineering
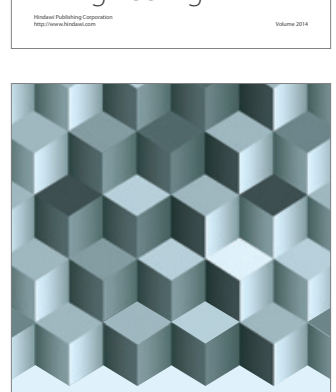

Journal of

Function Spaces
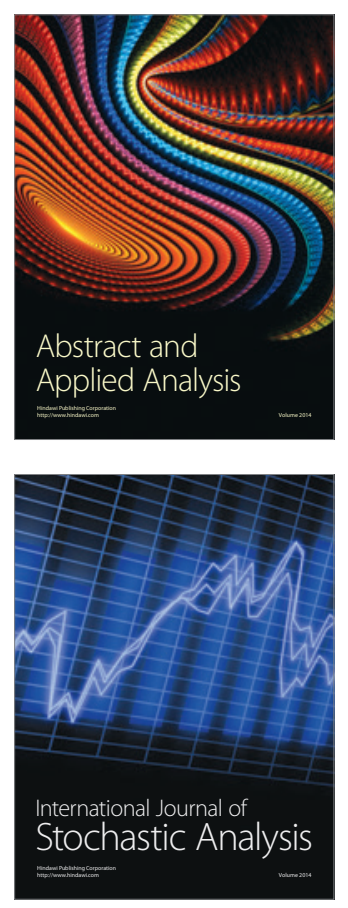

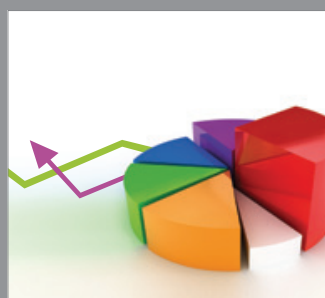

ournal of

Probability and Statistics

Promensencen
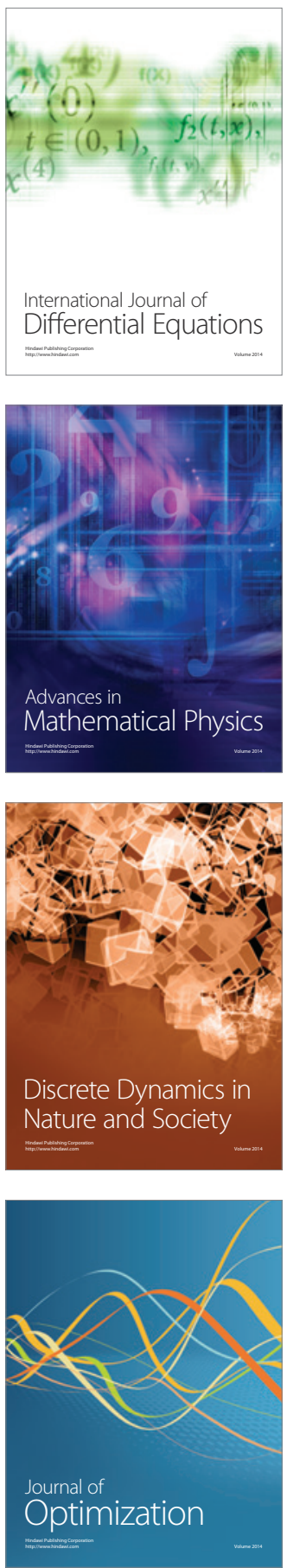